\title{
Computing Preferred Answer Sets by Meta-Interpretation in Answer Set Programming*
}

\author{
THOMAS EITER, WOLFGANG FABER \\ Institut für Informationssysteme 184/3, TU Wien \\ Favoritenstr. 9-11, 1040 Wien, Austria \\ (e-mail: $\{$ eiter, faber\}ekr.tuwien.ac.at) \\ NICOLA LEONE \\ Department of Mathematics, University of Calabria \\ 87030 Rende (CS), Italy \\ (e-mail: leone@unical.it) \\ GERALD PFEIFER \\ Institut für Informationssysteme 184/2, TU Wien \\ Favoritenstr. 9-11, 1040 Wien, Austria \\ (e-mail: pfeiferedbai.tuwien.ac.at)
}

\begin{abstract}
Most recently, Answer Set Programming (ASP) has been attracting interest as a new paradigm for problem solving. An important aspect, for which several approaches have been presented, is the handling of preferences between rules. In this paper, we consider the problem of implementing preference handling approaches by means of meta-interpreters in Answer Set Programming. In particular, we consider the preferred answer set approaches by Brewka and Eiter, by Delgrande, Schaub and Tompits, and by Wang, Zhou and Lin. We present suitable meta-interpreters for these semantics using DLV, which is an efficient engine for ASP. Moreover, we also present a meta-interpreter for the weakly preferred answer set approach by Brewka and Eiter, which uses the weak constraint feature of DLV as a tool for expressing and solving an underlying optimization problem. We also consider advanced meta-interpreters, which make use of graph-based characterizations and often allow for more efficient computations. Our approach shows the suitability of ASP in general and of DLV in particular for fast prototyping. This can be fruitfully exploited for experimenting with new languages and knowledge-representation formalisms.
\end{abstract}

\section{Introduction}

Handling preference information plays an important role in applications of knowledge representation and reasoning. In the context of logic programs and related formalisms, numerous approaches for adding preference information have been proposed, including

\footnotetext{
* This paper is a revised and extended version of a preliminary paper in: Alessandro Provetti and Tran Cao Son, editors, Proc. AAAI 2001 Spring Symposium on Answer Set Programming: Towards Efficient and Scalable Knowledge Representation and Reasoning, Stanford CA, March 2001, AAAI Press, ISBN 1-57735-129-0.
} 
(Baader and Hollunder 1995; Brewka 1994; Brewka 1996; Buccafurri et al. 1999; Delgrande and Schaub 2000; Gelfond and Son 1997; Marek and Truszczyński 1993; Rintanen 1998; Sakama and Inoue 2000; Zhang and Foo 1997). They have been designed for purposes such as capturing specificity or normative preference; see e.g. (Brewka and Eiter 1999; Delgrande and Schaub 2000; Sakama and Inoue 2000) for reviews and comparisons.

Answer Set Programming (ASP) is gaining importance as a new paradigm for (declarative) problem solving. Basically, ASP is function-free disjunctive logic programming with negation as failure and strong negation under the Answer Set Semantics (Gelfond and Lifschitz 1991). Several attempts have been made to combine ASP and preferential information, resulting in a number of different semantics for preferential ASP, such as those in (Brewka and Eiter 1999; Wang et al. 2000; Delgrande et al. 2000a), on which we will focus in this paper; (Schaub and Wang 2001) provides a unifying framework for them.

The following example is a classical situation for the use of preference information in non-monotonic reasoning.

Example 1 (bird \& penguin)

Consider the following logic program:

(1) penguin.

(2) bird.

(3) $\neg$ flies :- not flies, penguin.

(4) flies :- not $\neg$ flies, bird.

This program has two answers sets: $A_{1}=\{$ penguin, bird, $\neg$ flies $\}$ and $A_{2}=\{$ penguin, bird, flies $\}$, where we conclude $\neg$ flies and flies, respectively. Assume that rule $(i)$ has higher priority than $(j)$ iff $i<j$ (i.e., rule (1) has the highest priority and rule (4) the lowest). Then, $A_{2}$ is no longer intuitive, as flies is concluded from (4), which has lower priority than (3) where we conclude $\neg$ flies.

Interestingly, even if this example is very simple, various preference semantics arrive at different results. Furthermore, semantics which coincide on this example may well yield different results on other examples. Since evaluating a semantics on a number of benchmark examples, each of which possibly involving several rules, quickly becomes a tedious task, one would like to have a (quick) implementation of a semantics at hand, such that experimentation can be done using computer support. Exploring a (large) number of examples, which helps in assessing the behavior of a semantics, then can be performed significantly faster, easier, and less error prone than by manual evaluation.

In this paper, we address this issue and explore the implementation of preference semantics for logic programs by means of a powerful technique based on Answer Set Programming (ASP), which can be seen as a sort of meta-programming in ASP. There, a given logic program $\mathcal{P}$ with preferences is encoded by a suitable set of facts $F(\mathcal{P})$, which are added to a fixed "meta-program" $P_{I}$, such that the intended answer sets of $\mathcal{P}$ are determined by the answer sets of the logic program $P_{I} \cup F(\mathcal{P})$. The salient feature is that this $P_{I}$ is universal, i.e., it is the same for all input programs $\mathcal{P}$.

Pursuing such a meta-programming approach has several advantages: On the one hand, leveraging the underlying "programming" language as much as possible facilitates quick prototype implementations for the preference semantics at hand. On the other hand, this novel application of answer set programming improves the understanding of how the 
declarative nature of answer set semantics works, in that we see which methods are needed in order to express elements such as iterative and recursive definitions, respectively.

We recall that meta-interpretation is well-established in Prolog-style logic programming, but due to the differences in semantics, Prolog-style meta-interpreters noticeably differ from those presented in this paper. More specifically, Prolog-style meta-interpreters usually formalize resolution and its control, thereby exhibiting a rather procedural reading. Our ASP meta-interpreters, on the other hand, are formulated in a truly declarative way. This is more amenable to realizing interpreters for KR languages which have a purely declarative reading, and where syntactic elements such as the order of rules, or the order of literals in the body of a rule are not important.

However, the fundamental concept of ASP and Prolog-style meta-interpreters is the same: Representing programs using a formalism very close to the one they are written in, and providing a modular "interpreter" which evaluates the represented program by means of the common semantics, such that the results of the original and the interpreted programs are in correspondence. We will not go into further detail concerning the differences between Prolog-style and ASP-style meta-interpretation in this paper, but focus on presenting ASP-style meta-interpreters. It is worthwhile noting that meta-interpretation is not completely new in ASP; a similar technique was used previously in (Gelfond and Son 1997) for defining the semantics of logic programming with defeasible rules (cf. Section 6).

In this paper we focus on three similar, yet different semantics for prioritized logic programs, namely the preference semantics by Brewka and Eiter (1999), Wang, Zhou and Lin (2000), and Delgrande, Schaub and Tompits (2000a), which we refer to as B-preferred, W-preferred, and D-preferred answer set semantics, respectively. We present ASP metaprograms $P_{I_{B}}, P_{I_{W}}$, and $P_{I_{D}}$ such that the answer sets of $P_{I_{B}} \cup F(\mathcal{P}), P_{I_{W}} \cup F(\mathcal{P})$, and $P_{I_{D}} \cup F(\mathcal{P})$ correspond (modulo a simple projection function) precisely to the $\mathrm{B}-$, $\mathrm{W}-$-, and D-preferred answer sets of $\mathcal{P}$. This way, by running $F(\mathcal{P})$ together with the corresponding meta-program on the DLV system (Faber et al. 1999; Faber and Pfeifer 1996), we compute the preferred answer sets of $\mathcal{P}$ in a simple and elegant way. For B-preferred answer sets we also provide an alternate meta-program $P_{I_{g}}$, which implements a graph-based algorithm that deterministically checks preferredness of an answer set and, in general, is more efficient than $P_{I_{B}}$. Note that by suitable adaption of the meta-programs, other ASP engines such as Smodels (Niemelä et al. 2000) can be used as well.

The B-preferred answer set semantics refines previous approaches for adding preferences to default rules by Brewka (1994; 1996). It is defined for answer sets of extended logic programs (Gelfond and Lifschitz 1991) and is generalized to Reiter's default logic in (Brewka and Eiter 2000). An important aspect of this semantics is that the definition was guided by two general principles which, as argued, a preference semantics should satisfy. As shown in (Brewka and Eiter 1999), B-preferred answer sets satisfy these principles, while almost all other semantics do not. The $\mathrm{W}$ - and D-preferred answer set semantics increasingly strengthen the B-preferred answer set semantics (Schaub and Wang 2001).

Since, in general, programs having an answer set may lack a preferred answer set, in (Brewka and Eiter 1999) a relaxed notion, called weakly preferred answer sets, is defined. We implement this semantics by means of a meta-program $P_{I_{\text {weak }}}$, which takes advantage of the weak constraints feature (Buccafurri et al. 1997) of DLV.

Overall, the work reported in this paper is important in several respects: 
- We put forward the use of ASP for experimenting new semantics by means of a meta-interpretation technique. The declarativity of logic programs (LPs) provides a new and elegant way of writing meta-interpreters, which is very different from Prolog-style meta-interpretation. Thanks to the high expressiveness of (disjunctive) LPs and DLV's weak constraints, meta-interpreters can be written in a simple and declarative fashion.

- The description of the meta-programs implementing the various preference semantics also has a didactic value: it is a good example how meta-interpreters can be built using ASP. In particular, we also present a core meta-program for plain extended logic programs under answer set semantics, which may be used as a basic building block for the construction of other meta-programs.

- Furthermore, the meta-interpreters provide an actual implementation of preferred and weakly preferred answer sets and allow for easy experimentation of these semantics in practice. To our knowledge, this is the first implementation of weakly preferred answer sets. An implementation of preferred answer sets (also on top of DLV) has been reported in (Delgrande et al. 2000b), where programs are mapped into the framework of compiled preferences (Delgrande and Schaub 1997). Our implementation, as will be seen, is an immediate translation of the definition of preferred answer sets into DLV code. Weak constraints make the encoding of weakly preferred answer sets extremely simple and elegant, while that task would have been much more cumbersome otherwise. The meta-interpreters and a suite of example are available at http://www. dlvsystem. com/preferred/.

The experience reported in this paper confirms the benefits of ASP. It suggests the use of the DLV system as a high-level abstract machine to be employed also as a powerful tool for experimenting with new semantics and novel KR languages.

It is worthwhile noting that the meta-interpretation approach presented here does not aim at efficiency; rather, it fosters simple and very fast prototyping, which is useful e.g. in the process of designing and experimenting new languages.

The structure of the remainder of this paper is as follows: In the next section, we provide preliminaries of extended logic programming and answer set semantics. We then develop in Section 3 a basic meta-interpreter program for extended logic programs under the answer set semantics. After that, we consider in Section 4 meta-interpreter programs for B-preferred, W-preferred, and D-preferred answer answer sets. Section 5 is devoted to the refinement of preferred answer sets to weakly preferred answer sets. A discussion of related work is provided in Section 6. Finally, in Section 7 we summarize our results and draw some conclusions.

\section{Preliminaries: Logic Programs}

Syntax. Logic Programs (LPs) use a function-free first-order language. As for terms, strings starting with uppercase (resp., lowercase) letters denote variables (resp., constants). A (positive resp. negative) classical literal $l$ is either an atom $a$ or a negated atom $\neg a$, respectively; its complementary literal, denoted $\neg l$, is $\neg a$ and $a$, respectively. A (positive, resp. negative) negation as failure (NAF) literal $\ell$ is of the form $l$ or not $l$, where $l$ is a classical literal. Unless stated otherwise, by literal we mean a classical literal. 
A rule $r$ is a formula

$$
a_{1} \mathrm{v} \cdots \mathrm{v} a_{n}:-b_{1}, \cdots, b_{k}, \text { not } b_{k+1}, \cdots, \text { not } b_{m} .
$$

where all $a_{i}$ and $b_{j}$ are classical literals and $n \geq 0, m \geq k \geq 0$. The part to the left of ": -" is the head, the part to the right is the body of $r$; we omit ":-" if $m=0$. We let $H(r)=\left\{a_{1}, \ldots, a_{n}\right\}$ be the set of head literals and $B(r)=B^{+}(r) \cup B^{-}(r)$ the set of body literals, where $B^{+}(r)=\left\{b_{1}, \ldots, b_{k}\right\}$ and $B^{-}(r)=\left\{b_{k+1}, \ldots, b_{m}\right\}$ are the sets of positive and negative body literals, respectively. An integrity constraint is a rule where $n=0$.

A datalog program (LP) $P$ is a finite set of rules. We call $P$ positive, if $P$ is not -free (i.e. $\forall r \in P: B^{-}(r)=\emptyset$ ); and normal, if $P$ is $\mathrm{v}$-free (i.e. $\forall r \in P:|H(r)| \leq 1$ ).

A weak constraint $r$ is an expression of the form

$$
: \sim b_{1}, \cdots, b_{k}, \text { not } b_{k+1}, \cdots, \operatorname{not} b_{m} \cdot[w: l]
$$

where every $b_{i}$ is a literal, $l \geq 1$ is the priority level and $w \geq 1$ is the weight among the level. The intuition underlying levels and weights is that weak constraints of lower levels are more important than those of higher levels, and among the same level weak constraints with greater weights are more important. Both $l$ and $w$ are integers and set to 1 if omitted. The sets $B(r), B^{+}(r)$, and $B^{-}(r)$ are defined by viewing $r$ as an integrity constraint. $W C(P)$ denotes the set of weak constraints in $P$. Weak constraints are motivated by having a convenient means to deal with optimization problems such as the Traveling Salesperson problem, or express actions wit costs, cf. (Eiter et al. 2002).

As usual, a term (atom, rule,...) is ground, if no variables appear in it.

Semantics. Answer sets for LPs with weak constraints are defined by extending consistent answer sets for LPs as introduced in (Gelfond and Lifschitz 1991; Lifschitz 1996). We proceed in three steps: we first define answer sets (1) of ground positive programs, then (2) of arbitrary ground programs, and (3) finally (optimal) answer sets of ground programs with weak constraints. As usual, the (optimal) answer sets of a non-ground program $P$ are those of its ground instantiation $\operatorname{Ground}(P)$, defined below.

For any program $P$, let $U_{P}$ be its Herbrand universe and $B_{P}$ be the set of all classical ground literals from predicate symbols in $P$ over the constants of $U_{P}$; if no constant appears in $P$, an arbitrary constant is added to $U_{P}$. For any clause $r$, let $G r o u n d(r)$ denote the set of its ground instances. Then, $\operatorname{Ground}(P)=\bigcup_{r \in P} \operatorname{Ground}(r)$. Note that $P$ is ground iff $P=\operatorname{Ground}(P)$. An interpretation is any set $I \subseteq B_{P}$ of ground literals. It is consistent, if $I \cap\{\neg l \mid l \in I\}=\emptyset$.

In what follows, let $P$ be a ground program.

(1) A consistent ${ }^{1}$ interpretation $I \subseteq B_{P}$ is called closed under a positive program $P$, if $B(r) \subseteq I$ implies $H(r) \cap I \neq \emptyset$ for every $r \in P$. An interpretation $X$ is an answer set for $P$ if it is a minimal set (wrt. set inclusion) which is closed under $P$.

(2) Let $P^{I}$ be the Gelfond-Lifschitz reduct of a program $P$ w.r.t. $I \subseteq B_{P}$, i.e., the program obtained from $P$ by deleting

\footnotetext{
${ }^{1}$ We only consider consistent answer sets, while in (Lifschitz 1996) also the (inconsistent) set $B_{P}$ may be an answer set.
} 
- all rules $r \in P$ such that $B^{-}(r) \cap I \neq \emptyset$, and

- all negative body literals from the remaining rules.

Then, $I \subseteq B_{P}$ is an answer set of $P$ iff $I$ is an answer set of $P^{I}$. By $\mathcal{A S}(P)$ we denote the set of all answer sets of $P$.

\section{Example 2}

The program

$$
a \mathrm{v} b . \quad b \mathrm{v} c . \quad d \mathrm{v} \neg d \quad:-a, c .
$$

has three answer sets: $\{a, c, d\},\{a, c, \neg d\}$, and $\{b\}$.

(3) Given a program $P$ with weak constraints, we are interested in the answer sets of the part without weak constraints which minimize the sum of weights of the violated constraints in the highest priority level, and among them those which minimize the sum of weights of the violated constraints in the next lower level, etc. This is expressed by an objective function for $P$ and an answer set $A$ :

$$
\begin{aligned}
& f_{P}(1)=1 \\
& f_{P}(n)=f_{P}(n-1) \cdot|W C(P)| \cdot w_{\max }^{P}+1, \quad n>1 \\
& H_{A}^{P}=\sum_{i=1}^{l_{\max }^{P}}\left(f_{P}(i) \cdot \sum_{N \in N_{i}^{A, P}} w_{N}\right)
\end{aligned}
$$

where $w_{\max }^{P}$ and $l_{\max }^{P}$ denote the maximum weight and maximum level of a weak constraint in $P$, respectively; $N_{i}^{A, P}$ denotes the set of weak constraints in level $i$ which are violated by $A$, and $w_{N}$ denotes the weight of the weak constraint $N$.

Intuitively, $f_{P}(i)$ is a factor which guarantees that the impact of violated weak constraints of level $i$ is less than the impact of violated weak constraints of lower levels. More technically, note that $|W C(P)| \cdot w_{\max }^{P}+1$ is greater than the sum of all weights in the program, and is therefore guaranteed to be greater than any sum of weights of a single level.

Then, $A$ is an (optimal) answer set of $P$, if $A \in \mathcal{A S}(P \backslash W C(P))$ and $H_{A}^{P}$ is minimal over $\mathcal{A S}(P \backslash W C(P))$. Let $\mathcal{O} \mathcal{A S}(P)$ denote the set of all optimal answer sets.

\section{Example 3}

Let us extend the program from Example 2 by the following three weak constraints:

$$
: \sim a, c .[2: 1] \quad: \sim \neg d .[1: 1] \quad: \sim b .[3: 1]
$$

The resulting program $P_{3}$ has the single optimal answer set $A_{3}=\{a, c, d\}$ with weight 2 in level 1 .

ASP systems like DLV (and virtually all others) provide a built-in binary predicate which defines a total order on the constants. In DLV, this predicate is denoted $<$; in this paper, we denote it by $l t$, in order to avoid confusion with the rule ordering $<$ in programs which will be introduced in Section 4.

\section{Meta-Interpreting Answer Set Programs}

In this section we show how a normal propositional answer set program can be encoded for and interpreted by a generic meta-interpreter based on the following idea: 
We provide a representation $F(P)$ of an arbitrary normal propositional program $P^{2}$ as a set of facts and combine these facts with a generic answer set program $P_{I_{a}}$ such that $\mathcal{A S}(P)=\left\{\pi(A) \mid A \in \mathcal{A S}\left(F(P) \cup P_{I_{a}}\right)\right\}$, where $\pi$ is a simple projection function.

\subsection{Representing An Answer Set Program}

First we translate the propositional answer set program $P$ into a set of facts $F(P)$ as follows:

1. For each rule $c:-a_{1}, \ldots, a_{m}$, not $b_{1}, \ldots$, not $b_{n}$. of the program $P, F(P)$ contains the following facts:

$$
\operatorname{rule}(r) . \operatorname{head}(c, r) \cdot \operatorname{pbl}\left(a_{1}, r\right) \ldots \operatorname{pbl}\left(a_{m}, r\right) \cdot n b l\left(b_{1}, r\right) \ldots n b l\left(b_{n}, r\right) .
$$

where $r$ is a unique rule identifier.

2. For each pair of complementary literals $\ell$ and $\neg \ell$ occurring in the program $P$ we explicitly add a fact $\operatorname{compl}(\ell, \neg \ell)$. We denote $\neg \ell$ by $n e g_{-} \ell$ in programs.

\section{Example 4}

The program of the bird \& penguin example is represented by the following facts representing the rules and complementary literals:

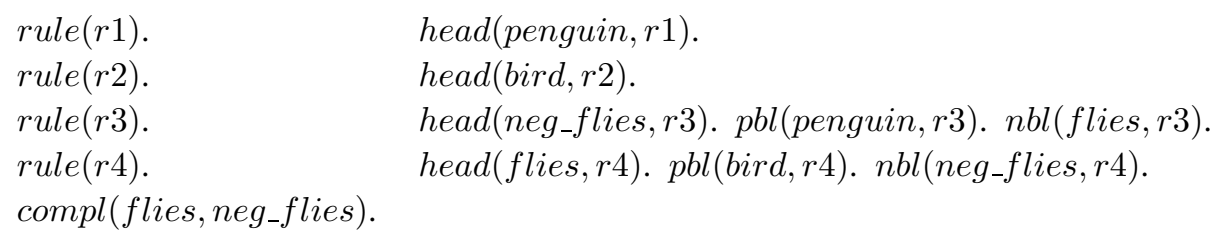

\subsection{Basic Meta-interpreter program}

Several meta-interpreters that we will present in the following sections consist of two parts: a meta-interpreter program $P_{I_{a}}$ for representing an answer set, and another one for checking preferredness. In this section we will provide the first part which is common to many meta-interpreters shown in this paper.

Representing an answer set. We define a predicate in_AS(.) which is true for the literals in an answer set of $P$. A literal is in an answer set if it occurs in the head of a rule whose positive body is definitely true and whose negative body is not false.

$$
i n_{-} A S(X):-h e a d(X, R), \text { pos_body_true }(R) \text {, not neg_body_false }(R) \text {. }
$$

The positive part of a body is true, if all of its literals are in the answer set. Unfortunately we cannot encode such a universal quantification in one rule. We can identify a simple case: If there are no positive body literals, the body is trivially true.

$$
\begin{aligned}
\text { pos_body_exists }(R) & :-\operatorname{pbl}(X, R) . \\
\text { pos_body_true }(R) & :-\operatorname{rule}(R), \operatorname{not} \text { pos_body_exists }(R) .
\end{aligned}
$$

\footnotetext{
${ }^{2}$ We assume that integrity constraints : $-C$. are written as equivalent rules bad: $-C$, not bad. where bad is a predicate not occurring in $P$.
} 
However, if positive body literals exist, we will proceed iteratively. To this end, we use the built-in constant ordering $l t$ for defining a successor relation on the positive body literals of each rule, and to identify the first and last literal, respectively, of a positive rule body in this total order. Technically, it is sufficient to define auxiliary relations as follows.

$$
\begin{aligned}
\text { pbl_inbetween }(X, Y, R) & :-\operatorname{pbl}(X, R), p b l(Y, R), p b l(Z, R), l t(X, Z), l t(Z, Y) . \\
\text { pbl_notmax }(X, R) & :-\operatorname{pbl}(X, R), p b l(Y, R), l t(X, Y) . \\
\text { pbl_notmin }(X, R) & :-\operatorname{pbl}(X, R), \operatorname{pbl}(Y, R), l t(Y, X) .
\end{aligned}
$$

This information can be used to define the notion of the positive body being true up to (w.r.t. the built-in order) some positive body literal. If the positive body is true up to the last literal, the whole positive body is true.

$$
\begin{aligned}
& \text { pos_body_true_upto }(R, X) \quad:-p b l(X, R), \operatorname{not} p b l \_n o t m i n(X, R), i n \_A S(X) \text {. } \\
& \text { pos_body_true_upto }(R, X) \quad:-\quad p o s \_b o d y \_t r u e \_u p t o(R, Y), p b l(X, R), i n \_A S(X) \text {, } \\
& l t(Y, X) \text {, not pbl_inbetween }(Y, X, R) \text {. } \\
& \text { pos_body_true }(R) \quad:-\quad \text { pos_body_true_upto }(R, X), \operatorname{not} p b l \_n o t m a x(X, R) \text {. }
\end{aligned}
$$

The negative part of a body is false, if one of its literals is in the answer set.

$$
n e g \_b o d y_{-} f a l s e(R) \quad:-n b l(X, R), i n \_A S(X) \text {. }
$$

Each answer set has to be consistent; we thus add an integrity constraint which rejects answer sets containing complementary literals.

$$
\text { :- } \operatorname{compl}(X, Y), i n_{-} A S(X), i n_{-} A S(Y) .
$$

The rules described above (referred to as $P_{I_{a}}$ in the sequel) are all we need for representing answer sets. Each answer set of $P_{I_{a}} \cup F(P)$ represents an answer set of $P$. Let $\pi$ be defined by $\pi(A)=\{\ell \mid$ in_AS( $\ell) \in A\}$. Then we can state the following:

\section{Theorem 1}

Let $P$ be a normal propositional program. Then, (i) if $A \in \mathcal{A S}\left(P_{I_{a}} \cup F(\mathcal{P})\right)$ then $\pi(A) \in$ $\mathcal{A S}(P)$, and (ii) for each $A \in \mathcal{A S}(P)$, there exists a single $A^{\prime} \in \mathcal{A S}\left(P_{I_{a}} \cup F(P)\right)$ such that $\pi\left(A^{\prime}\right)=A$.

\section{Proof}

(i) $\pi(A)$ must be a consistent set of literals from $P$, since for each $\ell$ s.t. $i n \_A S(\ell) \in A$, head $(\ell, r)$ must hold for some rule $r$, which, by construction of $F(P)$, only holds for $\ell \in B_{P}$, and since the constraint :- $\operatorname{compl}(\ell, \neg \ell), i n_{-} A S(\ell), i n_{-} A S(\neg \ell)$. must be be satisfied by $A$ (again by construction of $F(P)$ ) $\left\{i n \_A S(\ell), i n \_A S(\neg \ell)\right\} \nsubseteq A$ and hence $\{\ell, \neg \ell\} \nsubseteq \pi(A)$ for all $\ell \in B_{P}$.

Thus, to show that $\pi(A) \in \mathcal{A S}(P)$, it suffices to show that $(\alpha) \pi(A)$ is closed under $P^{\pi(A)}$, and that $(\beta) \pi(A) \subseteq T_{P^{\pi(A)}}^{\infty}$ must hold, where $T_{P^{\pi(A)}}$ is the standard $T_{P}$ operator for $P=P^{\pi(A)}$. Let, for convenience, denote $Q=\operatorname{Ground}\left(P_{I_{a}} \cup F(P)\right)$.

As for $(\alpha)$, we show that if $r \in P^{\pi(A)}$ such that $B(r) \subseteq \pi(A)$, then there is a rule $h_{r} \in$ $Q$ such that $H\left(h_{r}\right)=\left\{i n_{-} A S(h)\right\}$ where $H(r)=\{h\}$ and $h_{r}$ is applied in $A$, i.e., $A=$ $B\left(h_{r}\right)$ and $i n_{-} A S(h) \in A$. Let $r$ stem from a rule $r^{\prime} \in P$. Then, let $h_{r}$ be the (unique) rule in $Q$ such that $H\left(h_{r}\right)=\left\{i n_{-} A S(h)\right\}$ (note that $H(r)=H\left(r^{\prime}\right)=\{h\}$ ) and head $\left(h, r^{\prime}\right) \in$ $B\left(h_{r}\right)$. Since $B(r)=B^{+}\left(r^{\prime}\right) \subseteq \pi(A)$, we have for each $\ell \in B(r)$ that $i n \_A S(\ell) \in A$ and, 
by construction of $F(P)$, that $p b l\left(\ell, r^{\prime}\right) \in A$. Since $l t$ induces a linear ordering of $B^{+}\left(r^{\prime}\right)$, it follows by an inductive argument along it that pos_body_true_upto $\left(r^{\prime}, \ell\right) \in A$ holds for each $\ell \in B^{+}\left(r^{\prime}\right)$ and that pos_body_true $\left(r^{\prime}\right) \in A$. Furthermore, since $B^{-}\left(r^{\prime}\right) \cap \pi(A)=\emptyset$, it holds that $A \not \models B\left(n_{r}\right)$ for each rule $n_{r} \in Q$ such that $H\left(n_{r}\right)=n_{\text {eg_body_false }}\left(r^{\prime}\right)$. Therefore, neg_body_false $\left(r^{\prime}\right) \notin A$. Since head $\left(h, r^{\prime}\right) \in A$ by construction of $F(P)$, it follows that $A \models B\left(h_{r}\right)$. Thus, $h_{r}$ is applied in $A$, and hence $H\left(h_{r}\right)=i n_{-} A S(h) \in A$. This proves $(\alpha)$.

As for $(\beta)$, it suffices to show that if $i n_{-} A S(\ell) \in T_{Q^{A}}^{i} \backslash T_{Q^{A}}^{i-1}$, i.e., $i n_{-} A S(\ell)$ is added to $A$ in the $i$-th step of the least fixpoint iteration for $T_{Q^{A}}, i \geq 1$, then $\ell \in T_{P^{\pi(A)}}^{\infty}$ holds. Addition of $i n_{-} A S(\ell)$ implies that $\ell=H(r)$ for some $r \in P$ such that neg_body_false $(r) \notin A$ and pos_body_true $(r) \in A$. This implies in_AS( $\left.\ell^{\prime}\right) \in T_{Q^{A}}^{i-1}$ for each $\ell^{\prime} \in B^{+}(r)$ and in_AS $\left(\ell^{\prime}\right) \notin A$ for each $\ell^{\prime} \in B^{-}(r)$. By an inductive argument, we obtain $B^{+}(r) \subseteq$ $T_{P^{\pi(A)}}^{\infty} ;$ therefore, $\ell \in T_{P^{\pi(A)}}^{\infty}$ holds. This proves $(\beta)$.

(ii) For any $A \in \mathcal{A S}(P)$, let $A^{\prime}$ be defined as follows (lt is the total order on constants defined in $\mathrm{DLV})$ :

$$
\begin{aligned}
A^{\prime}= & F(P) \cup\{\text { in_AS }(x) \mid x \in A\} \cup\left\{\text { pos_body_true }(r) \mid \forall x \in B^{+}(r): x \in A\right\} \cup \\
& \left\{\text { pos_body_true_upto }(r, x) \mid x \in B^{+}(r) \wedge x \in A \wedge \forall y \in B^{+}(r): l t(y, x) \rightarrow y \in A\right\} \cup \\
& \left\{\text { neg_body_false }(r) \mid B^{-}(r) \cap A \neq \emptyset\right\} \cup\left\{\text { pos_body_exists }(r) \mid B^{+}(r) \neq \emptyset\right\} \cup \\
& \left\{p b l \_n o t m i n(x, r) \mid x \in B^{+}(r) \wedge \exists y \in B^{+}(r): l t(y, x)\right\} \cup \\
& \left\{p b l \_n o t m a x(x, r) \mid x \in B^{+}(r) \wedge \exists y \in B^{+}(r): l t(x, y)\right\} \cup \\
& \left\{p b l \_ \text {inbetween }(x, y, r) \mid x, y \in B^{+}(r) \wedge\left(\exists z \in B^{+}(r): l t(x, z) \wedge l t(z, y)\right)\right\}
\end{aligned}
$$

Observe that the set of literals defined by the five last sets (call it $A_{\text {stat }}$ ) do not depend on $A$ since they have to occur in all answer sets of $P_{I_{a}} \cup F(P)$. The definitions of $A_{\text {stat }}$ directly reflect the corresponding rule structure in $P_{I_{a}}$ and $F(P)$. Since the inclusion of literals $i n_{-} A S(x)$ into $A^{\prime}$ is determined by the condition $\pi\left(A^{\prime}\right)=A$, it is easy to see that all rules defining neg_body_false are satisfied; in the case of pos_body_true_upto and pos_body_true, rule satisfaction can be seen by a constructive argument along the order $l t$.

To see that $A^{\prime}$ is minimal and the only answer set of $P_{I_{a}} \cup F(P)$ s.t. $\pi\left(A^{\prime}\right)=A$, an argument similar to the one in $(\beta)$ of the proof for (i) can be applied: If another answer set $A^{\prime \prime}$ exists s.t. $\pi\left(A^{\prime \prime}\right)=A$, each $i n_{-} A S(x)$ s.t. $x \in A$ must be added in some stage of $T_{Q^{A^{\prime}}}^{i}$, and in some stage $T_{Q^{A^{\prime \prime}}}^{j}$ of the standard least fixpoint operator. But then, by an inductive argument, $T_{Q^{A^{\prime}}}^{\infty}=T_{Q^{A^{\prime \prime}}}^{\infty}$ must hold.

The meta-interpreter program $P_{I_{a}}$ has the benign property that a standard class of programs, namely the class of stratified programs, which are easy to evaluate, is also interpreted efficiently through it. Recall that a normal propositional program $P$ is stratified, if there is a function $\lambda$ which associates with each atom $a$ in $P$ an integer $\lambda(a) \geq 0$, such that each rule $r \in P$ with $H(r)=\{h\}$ satisfies $\lambda(h) \geq \lambda(\ell)$ for each $\ell \in B^{+}(r)$ and $\lambda(h)>\lambda(\ell)$ for each $\ell \in B^{-}(r)$. Denote for any program $P$ by $P_{i r}$ the set of rules in Ground $\left(P_{I_{a}} \cup F(P)\right)$ whose representation literals (i.e., literals over rule, head, $p b l, n b l$ ) are satisfied by $F(P)$. Then we have:

\section{Proposition 1}

Let $P$ be a stratified normal propositional program. Then, $P_{i r}$ is locally stratified (i.e., stratified if viewed as a propositional program). 
This can be seen by constructing from a stratification mapping $\lambda$ for $P$ a suitable stratification mapping $\lambda^{\prime}$ for $P_{i r}$. Locally stratified programs are handled efficiently by competitive ASP systems.

\section{Preferred Answer Sets}

In this section, we first introduce the underpinnings common to all three semantics for preferred answer sets we are considering in this paper and then provide the individual definitions.

\subsection{Prioritized Programs}

We recall and adapt the definitions of (Brewka and Eiter 1999) as needed in the current paper. Throughout the rest of this section, programs are tacitly assumed to be propositional.

Definition 1 (prioritized program)

A prioritized (propositional) program is a pair $\mathcal{P}=(P,<)$ where $P$ is a normal logic program without constraints, and $<$ is a strict partial order on $P$, i.e., an irreflexive ( $a \nless a$, for all $a$ ) and transitive relation.

Informally, $r_{1}<r_{2}$ means " $r_{1}$ has higher priority than $r_{2}$ ". For any $\mathcal{P}=(P,<)$, the answer sets of $\mathcal{P}$ are those of $P$; their collection is denoted by $\mathcal{A S}(\mathcal{P})=\mathcal{A S}(P)$.

\section{Definition 2 (full prioritization)}

A full prioritization of a prioritized program $\mathcal{P}=(P,<)$ is any $\left(P,<^{\prime}\right)$ where $<^{\prime}$ is a total order of $P$ refining $<$, i.e., $r_{1}<r_{2}$ implies $r_{1}<r_{2}$, for all $r_{1}, r_{2} \in P$. The set of all full prioritizations of $\mathcal{P}$ is denoted by $\mathcal{F} \mathcal{P}(\mathcal{P})$. We call $\mathcal{P}$ fully prioritized, if $\mathcal{F P}(\mathcal{P})=\{\mathcal{P}\}$.

\subsection{B-Preferred Answer Sets}

B-preferred answer sets have been introduced in (Brewka and Eiter 1999) as a refinement of previous approaches in (Brewka 1994; Brewka 1996). We first define B-preferred answer sets for a particular class of fully prioritized programs. A program $\mathcal{P}$ is prerequisite-free, if $B^{+}(r)=\emptyset$ for every rule $r \in \mathcal{P}$ holds. Furthermore, a literal $\ell$ (resp., a set $X$ of literals) defeats a rule $r$ of the form (1), if $\ell \in B^{-}(r)$ (resp., $X \cap B^{-}(r) \neq \emptyset$ ). A rule $r^{\prime}$ defeats a rule $r$ if $H\left(r^{\prime}\right)$ defeats $r$.

\section{Definition 3}

Let $\mathcal{P}=\left\{r_{1}, \ldots, r_{n}\right\}$ be a fully prioritized and prerequisite-free program. For any set $S \subseteq B_{P}$ of literals, the sequence $S_{i} \subseteq B_{P}(0 \leq i \leq n)$ is defined as follows:

$$
\begin{aligned}
& S_{0}=\emptyset \\
& S_{i}= \begin{cases}S_{i-1}, & \text { if }(\alpha) S_{i-1} \text { defeats } r_{i}, \text { or } \\
S_{i-1} \cup H\left(r_{i}\right), & (\beta) H\left(r_{i}\right) \subseteq S \text { and } S \text { defeats } r_{i},\end{cases}
\end{aligned}
$$

for all $i=1, \ldots, n$. The set $C_{\mathcal{P}}^{B}(S)$ is defined by 


$$
C_{\mathcal{P}}^{B}(S)= \begin{cases}S_{n}, & \text { if } S_{n} \text { is consistent } \\ B_{P} & \text { otherwise. }\end{cases}
$$

An answer set $A(=S)$ divides the rules of $P$ in Definition 3 into three groups: generating rules, which are applied and contribute in constructing $A$; dead rules, which are not applicable in $A$ but whose consequences would not add anything new if they were applied, since they appear in $A$; and zombies, which are the rules not applicable in $A$ whose consequences do not belong to $A$. Only zombies have the potential to render an answer set non-preferred. This is the case if some zombie is not "killed" by a generating rule of higher priority. If $A$ is a fixpoint of $C_{\mathcal{P}}^{B}$, then the inductive construction guarantees that indeed all zombies are defeated by generating rules with higher preference.

\section{Definition 4 (B-preferred answer set)}

Let $\mathcal{P}=(P,<)$ be a fully prioritized and prerequisite-free program, and let $A \in \mathcal{A S}(\mathcal{P})$. Then $A$ is a B-preferred answer set of $\mathcal{P}$ if and only if $C_{\mathcal{P}}^{B}(A)=A$.

In the case where $P$ is not prerequisite-free, a kind of dual Gelfond-Lifschitz reduct is computed as follows.

\section{Definition 5}

Let $\mathcal{P}=(P,<)$ be a fully prioritized program, and let $X \subseteq B_{P}$. Then ${ }^{X} \mathcal{P}=\left({ }^{X} P,{ }^{X}<\right)$ is the fully prioritized program such that:

- ${ }^{X} P$ is the set of rules obtained from $P$ by deleting

1. every $r \in \mathcal{P}$ such that $B^{+}(r) \nsubseteq X$, and

2. all positive body literals from the remaining rules.

- $X_{<}$is inherited from $<$by the map $f:{ }^{X} P \longrightarrow P$ (i.e., $r_{1}^{\prime} X_{<} r_{2}^{\prime}$ iff $f\left(r_{1}^{\prime}\right)<f\left(r_{2}^{\prime}\right)$ ), where $f\left(r^{\prime}\right)$ is the first rule in $P$ w.r.t. $<$ such that $r^{\prime}$ results from $r$ by Step 2.

The definition of $X_{<}$must respect possible clashes of rule priorities, as Step 2 may produce duplicate rules in general.

Definition 6 (B-preferred answer set (ctd.), $\mathcal{B P} \mathcal{A S})$

Let $\mathcal{P}=(P,<)$ be a prioritized program and $A \in A S(P)$. If $\mathcal{P}$ is fully prioritized, then $A$ is a B-preferred answer set of $\mathcal{P}$ iff $A$ is a B-preferred answer set of ${ }^{A} \mathcal{P}$; otherwise, $A$ is a B-preferred answer set of $\mathcal{P}$ iff $A$ is a B-preferred answer set for some $\mathcal{P}^{\prime} \in \mathcal{F} \mathcal{P}(\mathcal{P})$. By $\mathcal{B P} \mathcal{A S}(\mathcal{P})$ we denote the set of all B-preferred answer sets of $\mathcal{P}$.

\section{Example 5}

Reconsider the bird \& penguin example. Let us first check whether $A_{1}=\{$ penguin, bird, $\neg$ flies $\}$ is a B-preferred answer set. We determine the dual reduct ${ }^{A_{1}} \mathcal{P}$ which consists of the following rules:

$$
\begin{aligned}
& \text { (1) penguin. } \\
& \text { (2) } \text { bird. } \\
& \text { (3) } \quad \text { flies : }- \text { not flies. } \\
& \text { (4) } \quad \text { flies : }- \text { not } \neg \text { flies. }
\end{aligned}
$$


The order ${ }^{A_{1}}<$ coincides with $<$ as in Definition 5. Now, let us determine $A_{1,4}\left(=S_{4}\right)$, by constructing the sequence $A_{1, i}$, for $0 \leq i \leq 4: A_{1,0}=\emptyset, A_{1,1}=\{$ penguin $\}$, $A_{1,2}=\{$ penguin,bird $\}, A_{1,3}=$ \{penguin,bird, $\neg$ flies $\}$, and $A_{1,4}=A_{1,3}$. Thus, $A_{1,4}=\{$ penguin, bird, $\neg$ flies $\}=A_{1}$ and $C_{A_{1} \mathcal{P}}^{B}\left(A_{1}\right)=A_{1}$; hence, the answer set $A_{1}$ is preferred.

Next consider the answer set $A_{2}=\{$ penguin, bird, flies $\}$. The dual reducts ${ }^{A_{2}} \mathcal{P}$ and $A_{1} \mathcal{P}$ coincide, and thus $A_{2,4}=A_{1}$, which means $C_{A_{2} \mathcal{P}}^{B}\left(A_{2}\right) \neq A_{2}$. Hence, $A_{2}$ is not preferred, and $A_{1}$ is the single B-preferred answer set of $\mathcal{P}$.

The following example shows that not every prioritized program which has an answer set has also a B-preferred one.

\section{Example 6}

Consider the following program:

$$
\begin{aligned}
& \text { (1) } c:-\operatorname{not} b . \\
& \text { (2) } b:-\operatorname{not} a \text {. }
\end{aligned}
$$

where $(1)<(2)$. Its single answer set is $A=\{b\}$. However, $C_{A_{\mathcal{P}}}^{B}(A)=\{c, b\}$ and thus $A$ is not B-preferred. Indeed, no B-preferred answer set exists.

\subsubsection{Adapting the Meta-Interpreter}

Now we can extend the meta-interpreter for answer set programs from Section 3 to cover prioritized answer set programs.

Representing a prioritized program. A prioritized program $\mathcal{P}=(P,<)$ is represented by a set of facts $F(\mathcal{P})$ which contains $F(P)$ plus, for each rule preference $r<r^{\prime}$ that belongs to the transitive reduction of $<$, a fact $\operatorname{pr}\left(r, r^{\prime}\right)$.

\section{Example 7}

In the case of our bird \& penguin example, we add the following three facts:

$$
\operatorname{pr}(r 1, r 2) . \quad \operatorname{pr}(r 2, r 3) . \quad \operatorname{pr}(r 3, r 4) .
$$

Checking preferredness. According to Definition 6, we have to create all fully prioritized programs $\mathcal{F} \mathcal{P}(\mathcal{P})$ of $\mathcal{P}$ to determine its preferred answer sets. To this end, we add code to guess a total order on the rules, refining $<$ :

$$
\begin{aligned}
\operatorname{pr}(X, Y) \mathrm{v} p r(Y, X) & :-\operatorname{rule}(X), \operatorname{rule}(Y), X !=Y . \\
\operatorname{pr}(X, Z) & :-\operatorname{pr}(X, Y), \operatorname{pr}(Y, Z) . \\
& :-\operatorname{pr}(X, X) .
\end{aligned}
$$

The rules state the axioms of totality, transitivity, and irreflexivity of a total order. Note that it would be possible to replace the disjunctive guessing rule by two rules involving unstratified negation. However, the disjunctive version is more readable.

Next we build the set $C_{\mathcal{P}^{\prime}}^{B}$ from Definition 3 where $\mathcal{P}^{\prime}={ }^{X} \mathcal{P}$. To this end, we do not compute the sets $S_{i}$ as in Definition 3 - clearly one rule can contribute at most one element to $C_{\mathcal{P}^{\prime}}^{B}$ and we represent this fact using the predicate $l i t(.,$.$) . We first observe that duplicate$ 
rules arising in the dual reduct $\mathcal{P}$ need no special care, since only the first occurrence of a rule from $\mathcal{P}^{\prime}$ is relevant for the value of $C_{\mathcal{P}^{\prime}}^{B}$; for later occurrences of duplicates $S_{i}=S_{i-1}$ will always hold.

In Definition 3, a condition is stated, which holds whenever $H\left(r_{i}\right)$ is not added, while $\operatorname{lit}(.,$.$) represents the opposite, so we negate the condition: (\beta)$ is actually itself a conjunction $\gamma \wedge \delta$, so the condition we are interested in is

$$
\neg(\alpha \vee(\gamma \wedge \delta)) \equiv(\neg \alpha \wedge \neg \gamma) \vee(\neg \alpha \wedge \neg \delta) .
$$

We call condition $\alpha$ local defeat (by rules of higher priority) and $\delta$ global defeat (by the answer set).

Definition 3 applies only to prerequisite-free programs, so for the general case we also have to include the definition of the dual Gelfond-Lifschitz reduct, which amounts to stating that only rules with a true positive body w.r.t. the answer set have to be considered. The encoding is then straightforward:

$$
\begin{aligned}
\operatorname{lit}(X, Y):- & h e a d(X, Y), \text { pos_body_true }(Y), \\
& \operatorname{not} d e f e a t \_l o c a l(Y), \operatorname{not} i n \_A S(X) . \\
\operatorname{lit}(X, Y):- & h e a d(X, Y), \operatorname{pos} \_b o d y \_t r u e(Y), \\
& \operatorname{not} d e f e a t \_l o c a l(Y), \operatorname{not} d e f e a t \_g l o b a l(Y) . \\
\text { defeat_local }(Y):- & n b l(X, Y), \operatorname{lit}(X, Y 1), \operatorname{pr}(Y 1, Y) . \\
\text { defeat_global }(Y):- & n b l(X, Y), \text { in_AS }(X) .
\end{aligned}
$$

The set $C_{\mathcal{P}^{\prime}}^{B}$ is the union of all literals in $\operatorname{lit}(.,$.$) :$

$$
i n_{-} C P(X):-\operatorname{lit}(X, Y) \text {. }
$$

Finally, according to Definition 4, a preferred answer set $A$ must satisfy $A=C_{\mathcal{P}^{\prime}}^{B}(A)$, so we formulate integrity constraints which discard answer sets violating this condition:

$$
\begin{aligned}
& :-\quad i n_{-} C P(X), \text { not } i n_{-} A S(X) . \\
& :-\quad i n_{-} A S(X), \text { not } i n_{-} C P(X) .
\end{aligned}
$$

This completes the meta-interpreter program $P_{I_{B}}$. A compact listing of it (without showing $P_{I_{a}}$ explicitly) is given in Figure 1 . The following result states that it works correctly.

\section{Theorem 2}

Let $\mathcal{P}=(P,<)$ be a propositional prioritized program. Then, (i) if $A \in \mathcal{A S}\left(P_{I_{B}} \cup\right.$ $F(\mathcal{P})$ ) then $\pi(A) \in \mathcal{B P} \mathcal{A S}(\mathcal{P})$, and (ii) for each $A \in \mathcal{B P} \mathcal{A S}(\mathcal{P})$, there exists some $A^{\prime} \in \mathcal{A S}\left(P_{I_{B}} \cup F(\mathcal{P})\right)$ such that $\pi\left(A^{\prime}\right)=A$.

\section{Proof}

Let $Q=P_{I_{B}} \cup F(\mathcal{P})$ and $Q_{a}=P_{I_{a}} \cup F(\mathcal{P})$. By well-known results about splitting a logic program (Lifschitz and Turner 1994), we obtain that for each answer set $A$ of $Q$, its restriction $A_{a}$ to the predicates of $Q_{a}$ is an answer set of $Q_{a}$, and that $A$ is an answer set of $\left(P_{I_{B}} \backslash Q_{a}\right) \cup A_{a}$.

(i) Suppose that $A \in \mathcal{A S}(Q)$. Then, $A_{a}$ is an answer set of $Q_{a}$, and thus, by Theorem 1, $S=\pi\left(A_{a}\right)(=\pi(A))$ is an answer set of $P$. Furthermore, by the three clauses that define and constrain the predicate $p r$ in $P_{I_{B}}, A$ defines a total ordering $<^{\prime}$ on $P$ such that $r<^{\prime} r^{\prime}$ 


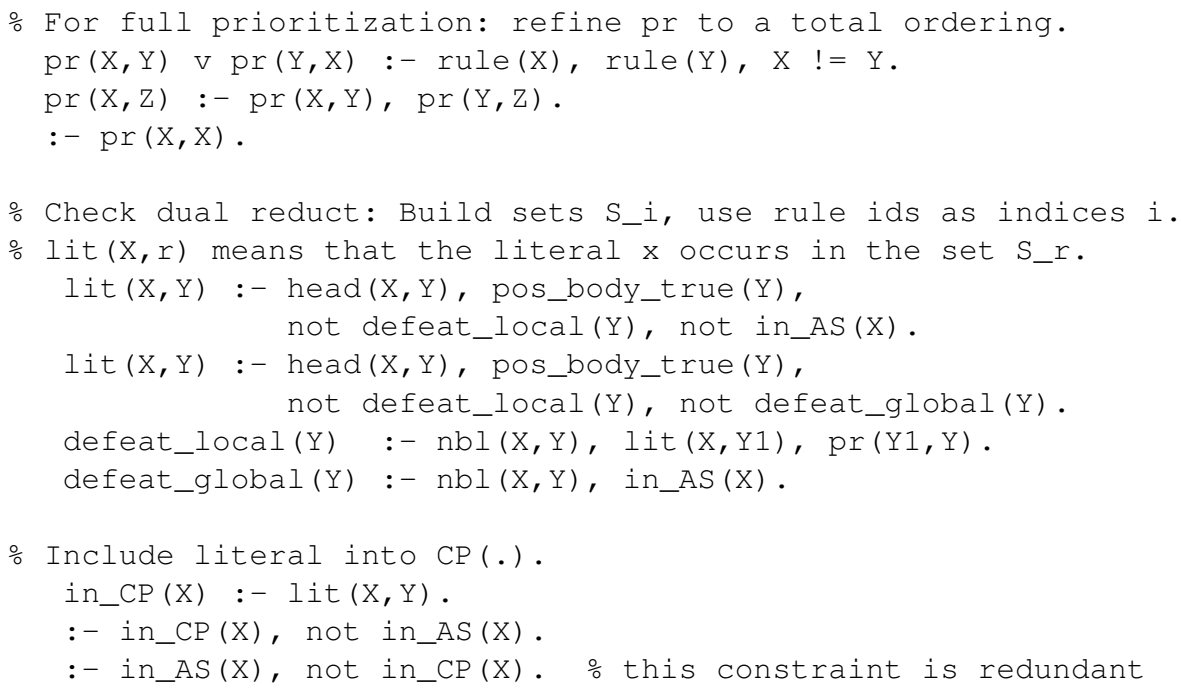

Fig. 1. Meta-Interpreter $P_{I_{B}}$ for B-Preferred Answer Sets (without $P_{I_{a}}$ )

is equivalent to $\operatorname{pr}\left(r, r^{\prime}\right)$. Consider the dual reduct ${ }^{S} \mathcal{P}=\left({ }^{S} P,{ }^{S}<\prime\right)$. Since $S$ is an answer set of $P$, it remains to show that $C_{\mathcal{S P}_{\mathcal{P}}}^{B}(S)=S$ holds. Define the sets $S_{i}, 0 \leq i \leq n$, along the ordering ${ }^{S}<^{\prime}$ as follows. Denote by $r_{i}^{S_{\mathcal{P}}} \in P$ the least rule $r$ under $<^{\prime}$ such that its dual reduct ${ }^{S} r$ (i.e., $H\left({ }^{S} r\right)=H(r)$ and $\left.B\left({ }^{S} r\right)=B^{-}(r)\right)$ is the $i$-th rule in ${ }^{S} \mathcal{P}$ under ${ }^{S}<^{\prime}$, where $1 \leq i \leq n$. Then, let $S_{0}=\emptyset$ and let $S_{i}=\left\{\ell \mid \operatorname{lit}(\ell, r) \in A\right.$, where $r=r_{j}^{S_{\mathcal{P}}}$ for some $j \leq i\}$, for all $i=1, \ldots, n$.

By an inductive argument on $i=0, \ldots, n$, we obtain that the sequence $S_{0}, \ldots, S_{n}$ satisfies the condition of Definition 3. Indeed, this is true for $i=0$. Suppose it is true for $i-1$ and consider $i$. Let $r=r_{i}^{S_{\mathcal{P}}}$ where $H(r)=\{h\}$. Then, pos_body_true $(r)$ $\in A$ holds. If $h \in S_{i-1}$ holds, then the condition in Definition 3 anyway holds. Thus, suppose $h \notin S_{i-1}$ and first that $S_{i}=S_{i-1}$. Then, both rules with head $l i t(h, r)$ in $Q$ are not applied in $A$. This means that either $(\alpha)$ defeat_local $(r) \in A$ or $(\beta)\left\{i n \_A S(h)\right.$, defeat_global $(r)\} \subseteq A$ must hold. Case $(\alpha)$ implies that, by the induction hypothesis, there is some literal $\ell \in B^{-}(r)$ such that $\ell \in S_{i-1}$; that is, $S_{i-1}$ defeats $r$. Case $(\beta)$ means that $H(r) \subseteq S$ and that $S$ defeats $r$. Thus, $S_{i}$ satisfies Definition 3 in this case.

Otherwise, suppose we have $h \in S_{i} \backslash S_{i-1}$. Thus, $\operatorname{lit}(h, r) \in A$, which means that one of the two rules with head $l i t(h, r)$ in $Q$ is applied in $A$. Thus, we have $(\alpha)$ defeat_local $(r) \notin$ $A$, which, by the induction hypothesis, means that $r$ is not defeated by $S_{i-1}$, and that $(\beta)$ either $i n \_A S(h) \notin A$, hence $H(r) \nsubseteq S$, or defeat_global $(r) \notin A$, which, by the definition of defeat_global, means that $S$ does not defeat $r$. Thus, the condition of Definition 3 is satisfied. This proves the claim on the sequence $S_{0}, \ldots, S_{n}$.

As easily seen, $S_{n}=\{\ell \mid \exists r:$ lit $(\ell, r) \in A\}$. Therefore, from the rule defining in_CP in $P_{I_{B}}$, we obtain that $i n_{-} C P(\ell) \in A$ holds iff $\ell \in S_{n}$, for any constant $\ell$. Thus, from the last two constraints of $P_{I_{B}}$, we infer that $C_{S \mathcal{P}}^{B}(S)=S$ must hold; in other words, $S=\pi(A)$ is a $B$-preferred answer set of $\mathcal{P}$. 
(ii) Suppose that $A \in \mathcal{B P} \mathcal{A} \mathcal{S}(\mathcal{P})$, and let $\mathcal{P}^{\prime}=\left(P,<^{\prime}\right)$ be a full prioritization of $\mathcal{P}$, such that $A \in \mathcal{B P} \mathcal{A S}\left(\mathcal{P}^{\prime}\right)$. Then, we obtain an answer set $A^{\prime}$ of $Q$ as follows:

- On the predicates defined in $P_{I_{a}} \cup F(\mathcal{P}), A^{\prime}$ coincides with the answer set of $P_{I_{a}} \cup$ $F(P)$ corresponding to $A$ as in item (ii) of Theorem 1;

- $p r$ is defined according to $<^{\prime}$, i.e., $p r\left(r, r^{\prime}\right)$ iff $r<^{\prime} r^{\prime}$;

- $\operatorname{lit}(h, r)$ is true iff $r$ is a rule from $P$ such that $r$ is applied in $A$;

- defeat_local $(r)$ is true iff some rule $r^{\prime}<^{\prime} r$ exists such that $H\left(r^{\prime}\right) \cap B^{-}(r) \neq \emptyset$ and $r^{\prime}$ is applied in $A$;

- defeat_global $(r)$ is true iff $A \cap B^{-}(r) \neq \emptyset$;

- $i n_{-} C P(\ell)$ is true iff $\ell \in A$.

Note that $A^{\prime}$ satisfies the last two constraints in $P_{I_{B}}$ by virtue of Theorem 1 , since $A$ is an answer set of $\mathcal{P}$.

By the splitting result of (Lifschitz and Turner 1994), for showing that $A^{\prime}$ is an answer set of $Q$, we only need to show the following. Let $A^{\prime \prime}$ be the restriction of $A^{\prime}$ to the predicates in $P_{I_{a}} \cup F(\mathcal{P})$ and $p r$; then, $A^{\prime}$ is an answer set of the program $Q_{1}$, which contains $A^{\prime \prime}$ plus all clauses $c$ in $Q$ which involve the predicates lit, defeat_local, defeat_global, in_CP and such that $A^{\prime \prime}$ satisfies all literals in $B(c)$ on the predicates in $P_{I_{a}}$ and $p r$.

As easily seen, $Q_{1}$ is locally stratified, and a stratification $\lambda$ exists on the atoms occurring in $Q_{1}$ such that

- $\lambda\left(\right.$ lit $\left.\left(\ell^{\prime}, r^{\prime}\right)\right)<\lambda($ lit $(\ell, r))$ and $\lambda\left(\right.$ defeat_local $\left.\left(r^{\prime}\right)\right)<\lambda($ defeat_local $(r))$, for all constants $\ell, \ell^{\prime}$ and rules $r, r^{\prime} \in P$ such that $r^{\prime}<{ }^{\prime} r$;

- $\lambda($ defeat_local $(r))<\lambda($ lit $(\ell, r))$, for all literals $\ell$ and rules $r \in P$;

- $\lambda($ in_CP $C(\ell))=1+\max \left\{\lambda(\right.$ lit $(\ell, r)) \mid$ head $\left.(\ell, r) \in A^{\prime \prime}\right\} ;$ and

- $\lambda(a)=0$, for all other atoms $a$ in $Q_{1}$.

Then, along $\lambda$, we can verify that $Q_{1}$ has a stratified model $S$ which coincides with $A^{\prime}$, i.e., for $i \geq 0$, we have for all atoms $a$ with $\lambda(a) \leq i$ that $a \in A^{\prime}$ iff $a \in S$.

For $i=0$, this is immediate from the definition of $A^{\prime}$. Suppose the statement is true for $i$, and consider $i+1$. Let $a$ be an atom such that $\lambda(a)=i+1$. Suppose first that $a \in A^{\prime}$, and consider the possible cases:

- If $a=$ defeat_local $(r)$, then some $r^{\prime}<^{\prime} r$ exists such that $H\left(r^{\prime}\right) \cap B^{-}(r) \neq \emptyset$ and $r^{\prime}$ is applied in $A$. By definition, $\operatorname{lit}\left(\ell, r^{\prime}\right) \in A^{\prime}$ holds, and by the induction hypothesis, lit $\left(\ell, r^{\prime}\right) \in S$. Thus, defeat_local $(r) \in S$.

- Next, if $a=i n \_C P(\ell)$, then by definition of $A^{\prime}, \ell \in A$. Since $A$ is a $B$-preferred answer set of $\mathcal{P}^{\prime}$, it follows that $l i t(\ell, r) \in A^{\prime}$ for some $r$ such that $\lambda($ lit $(\ell, r)) \leq i$. Thus, by the induction hypothesis, $l i t(\ell, r) \in S$, which implies $i n_{-} C P(\ell) \in S$.

- Finally, if $a=\operatorname{lit}(\ell, r)$, then by definition of $A^{\prime}, r$ is applied in $A$. Thus, we have by construction head $(\ell, r) \in S$, pos_body_true $(r) \in S$, defeat_global $(r) \notin S$, and all these atoms rank lower than $l i t(\ell, r)$. Furthermore, de feat local $(r) \notin S$ must hold; otherwise, as $\lambda($ defeat_local $(r))<\lambda($ lit $(\ell, r))$, by the induction hypothesis, some rule $r^{\prime}<^{\prime} r$ would exist which is applied in $A$ such that $H\left(r^{\prime}\right) \cap B^{-}(r) \neq \emptyset$, which would contradict that $r$ is applied in $A$. This means, however, that the second rule with head lit $(\ell, r)$ in $Q_{1}$ is applied, and thus $l i t(\ell, r) \in S$. 
Thus, $a \in A^{\prime}$ implies $a \in S$. Conversely, suppose that $a \in S$, and again consider the possible cases:

- If $a=$ defeat_local $(r)$, then it follows that $\operatorname{lit}\left(\ell, r^{\prime}\right) \in S$ for some $r^{\prime}<^{\prime} r$ such that $H\left(r^{\prime}\right) \cap B^{-}(r) \neq \emptyset$. By the induction hypothesis, $\operatorname{lit}\left(\ell, r^{\prime}\right) \in A^{\prime}$, which by definition of $A^{\prime}$ means that $r^{\prime}$ is applied in $A$; since $H\left(r^{\prime}\right) \cap B^{-}(r) \neq \emptyset$, by definition of $A^{\prime}$ we have defeat_local $(r) \in A^{\prime}$.

- Next, if $a=i n_{-} C P(\ell)$, then $\operatorname{lit}(\ell, r) \in S$ exists such that $\lambda(\operatorname{lit}(\ell, r)) \leq i$. By the induction hypothesis and the definition of $A^{\prime}$, we have that $r$ is applied in $A$. Therefore, $\ell \in A$, which by definition means $i n_{-} C P(\ell) \in A^{\prime}$.

- Finally, if $a=l i t(\ell, r)$, then head $(\ell, r) \in A^{\prime}$, pos_body_true $(r) \in A^{\prime}$, defeat_local $(r) \notin$ $A^{\prime}$, and either $(\alpha)$ in_AS( $(\ell) \notin A^{\prime}$, or $(\beta)$ defeat_global $(r) \notin A^{\prime}$. In case $(\alpha)$, by definition of $A^{\prime}$ we have $\ell \notin A$. This implies, however, that $A$ is not a $B$-preferred answer set of $\mathcal{P}$, which is a contradiction. Thus, $(\beta)$ must apply. By the induction hypothesis, we obtain defeat_global $(r) \notin S$. Hence, $B^{-}(r) \cap A=\emptyset$, which means that $r$ is applied in $A$; hence, $\operatorname{lit}(\ell, r) \in A^{\prime}$ by definition.

This shows that $a \in S$ implies $a \in A^{\prime}$, which concludes the induction. We thus have shown that $S=A^{\prime}$. Since $A^{\prime}$ satisfies the last two constraints of $P_{I_{B}}$, it follows that $S$ is the stratified model of $Q_{1}$ and $A^{\prime}$ is an answer set $Q_{1}$. Hence, $A^{\prime}$ is an answer set of $Q$. This proves the result.

\section{Example 8}

For the bird \& penguin example, $P_{I_{B}} \cup F(\mathcal{P})$ has one answer set, containing in_AS(bird), in_AS(penguin), and in_AS( $\neg$ flies $)$.

We note that the last constraint in $P_{I_{B}}$ is in fact redundant and can be dropped; this is possible since the fixpoint condition $C_{\mathcal{P}}^{B}(A)=A$ in the Definition 4 can be equivalently replaced by a weaker condition as follows.

\section{Proposition 2}

Let $\mathcal{P}=(P,<)$ be a fully prioritized and prerequisite-free program, and let $A \in \mathcal{A S}(\mathcal{P})$. Then, $A \in \mathcal{B P} \mathcal{A S}(\mathcal{P})$ iff $C_{\mathcal{P}}^{B}(A) \subseteq A$.

Proof

It suffices to show that $C_{\mathcal{P}}^{B}(A) \subseteq A \wedge C_{\mathcal{P}}^{B}(A) \neq A$ raises a contradiction. Assume that the condition holds. Then, some $\ell \in A \backslash C_{\mathcal{P}}^{B}(A)$ must exist, which means that a generating rule $r$ w.r.t. $A$ must exist such that $H(r)=\{\ell\}$ and $A=B(r)$. According to Definition 3, $(\alpha) \vee(\beta)$ must hold for $A_{r}$, otherwise $\ell \in C_{\mathcal{P}}^{B}(A)$ would hold. Now $(\beta)$ cannot hold, since $A$ cannot defeat $r$ because $A \models B(r)$. Thus $(\alpha)$ must hold. This implies that some $\ell^{\prime} \in A_{r-1}$ defeats $r$ such that $\ell^{\prime} \notin A$. Since $A_{r-1} \subseteq C_{\mathcal{P}}^{B}(A), C_{\mathcal{P}}^{B}(A) \nsubseteq A$ follows. This is a contradiction.

\subsubsection{Deterministic Preferredness Checking}

The method we provided above non-deterministically generates, given a prioritized program $\mathcal{P}=(P,<)$ and an answer set of $\mathcal{P}$, all full prioritizations of $\mathcal{P}$ and tests them.

In (Brewka and Eiter 1999) a graph-based algorithm has been described which checks 
preferredness of an answer set $A$ deterministically without refining $<$ to a total order. In general, this method is much more efficient.

This approach works as follows: A labeled directed graph $G(\mathcal{P}, A)$ is constructed, whose vertices are the rules in $P$, and an edge leads from $r$ to $r^{\prime}$ if $r<r^{\prime}$. Each vertex $r$ is labeled "g" if $r$ is generating w.r.t $A$, " $z$ " if it is a zombie, and " $\mathrm{i}$ " (for irrelevant) otherwise. The following algorithm then performs a kind of topological sorting for deciding whether an answer set $A$ is preferred, and outputs a suitable full prioritization of $\mathcal{P}$ :

\section{Algorithm FULL-ORDER}

Input: A propositional prioritized program $\mathcal{P}=(P,<)$, and an answer set $A \in \mathcal{A S}(P)$.

Output: A full prioritization $\mathcal{P}^{\prime} \in \mathcal{F} \mathcal{P}(\mathcal{P})$ such that $A \in \mathcal{B P} \mathcal{A S}\left(\mathcal{P}^{\prime}\right)$ if $A \in \mathcal{B P} \mathcal{A S}(\mathcal{P})$; "no", otherwise.

\section{Method:}

Step 1. Construct the graph $G=G(\mathcal{P}, A)$, and initialize $S:=\emptyset,<^{\prime}:=\emptyset$.

Step 2. If $G$ is empty, then output $\mathcal{P}^{\prime}=\left(P,<^{\prime}\right)$ and halt.

Step 3. Pick any source of $G$, i.e., a vertex $r$ with no incoming edge, such that either $r$ is not labeled "z" or $r$ is defeated by $S$. If no such $r$ exists, then output "no" and halt.

Step 4. If $r$ is labeled "g", then set $S:=S \cup H(r)$.

Step 5. Remove $r$ from $G$, and continue at Step 2.

A discussion of this algorithm is given in (Brewka and Eiter 1999). Note that it is nondeterministic in Step 3. A deterministic variant of it can be used for merely deciding preferredness of $A$ : rather than some arbitrary source $r$, all sources $r$ satisfying the condition are selected in Step 3 and then removed in parallel in Step 5. As easily seen, this is feasible since removability of a source $r$ is monotone, i.e., can not be invalidated by removing any other source $r^{\prime}$ before. $A$ is a preferred answer set iff the algorithm stops with the empty graph, i.e., all vertices are removed.

This deterministic algorithm can be readily encoded in DLV. The idea is to use stages for modeling the iterations through Steps $2-5$. Since the number of steps is bounded by the number of rules in $\mathcal{P}$, it is possible to avoid introducing new symbols for these stages and reuse the rule-IDs for this purpose instead:

$$
\operatorname{stage}(T):-\operatorname{rule}(T) .
$$

Similar as in Section 3.2, we employ the built-in total order $l t$ on constants for ordering the stages. For example, if we have rule $(r 1)$ and rule $(s 1)$, then we have stage $(r 1)$ and stage $(s 1)$, and $r 1$ is "earlier" than $s 1$ since $l t(r 1, s 1)$ holds in DLV's built-in order $l t$. It is worthwhile noting that any total order on the stage symbols would work, and that this ordering is different from the ordering of the rules given in the input (which is partial in general). The first (least) stage is used for the stage after the first run through Steps 2-5.

We use predicates $g$ and $z$ for rule labels "g" and " $z$ ", respectively, which are defined as follows (label "i" is not of interest and thus omitted):

$$
\begin{aligned}
& g(R):- \text { rule }(R), \text { pos_body_true }(R), \text { not neg_body_false }(R) . \\
& z(R) \quad:- \text { rule }(R), \text { pos_body_true }(R), \text { head }(X, R), \text { not in_AS }(X) .
\end{aligned}
$$

Initially, only sources which are not zombies can be removed from the graph. We use a predicate nosource $0(R)$, which informally means that $R$ is not a source node in $G$, and a predicate remove $(R, S)$ which means that at stage $S$, the vertex $R$ is no longer in $G$ : 


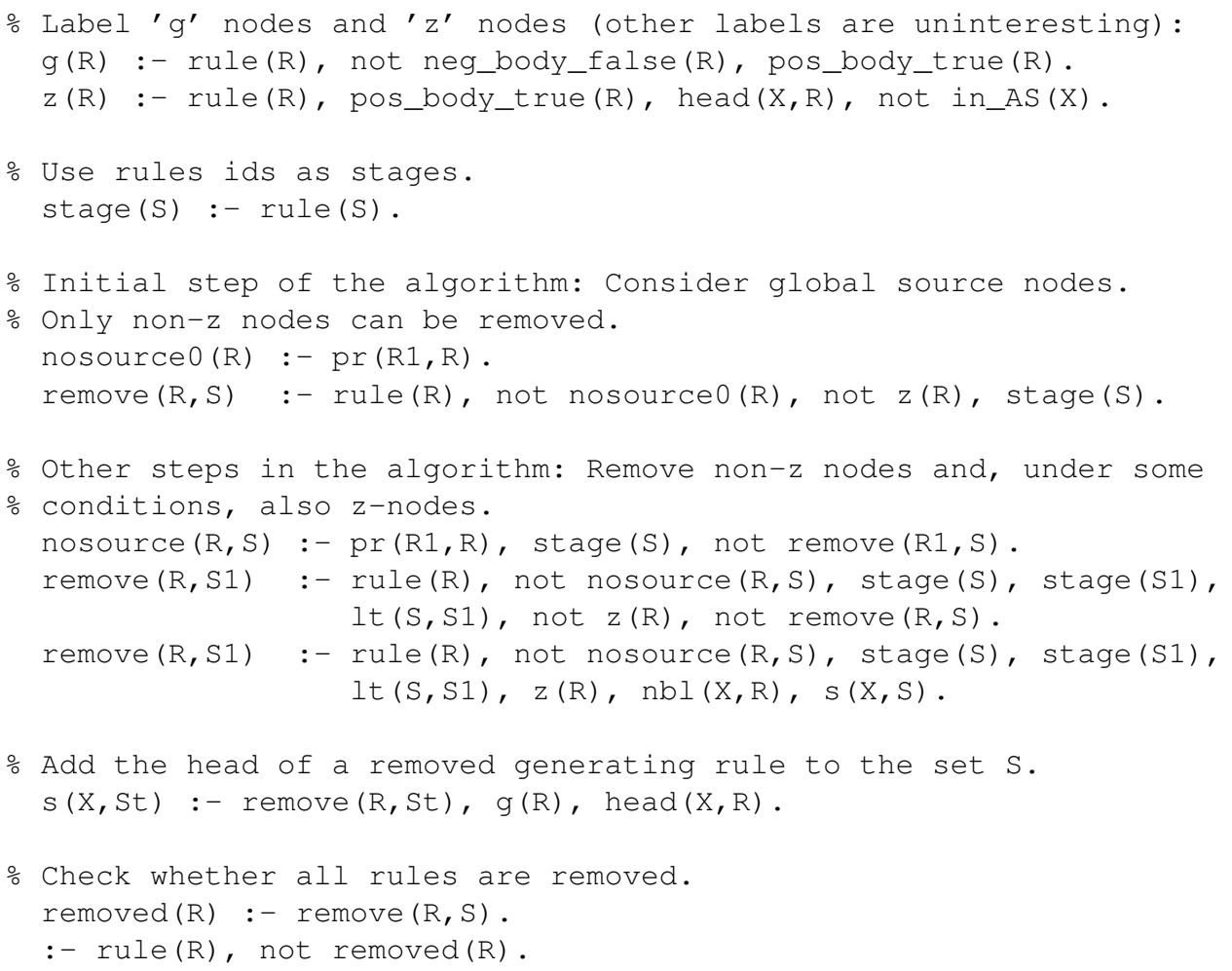

Fig. 2. Meta-Interpreter $P_{I_{g}}$ for B-Preferred Answer Sets Using Deterministic Preferredness Checking (without $P_{I_{a}}$ )

$$
\begin{aligned}
& \operatorname{nosource} 0(R) \quad:-\operatorname{pr}(R 1, R) \text {. } \\
& \text { remove }(R, S) \text { :- rule }(R) \text {, not nosource }(R), \text { not } z(R), \text { stage }(S) \text {. }
\end{aligned}
$$

At other stages of the iteration, we can remove all rules satisfying the condition of Step 3. We use a predicate nosource $(R, S)$ which expresses that $R$ is not a source at stage $S$.

$$
\begin{aligned}
\operatorname{nosource}(R, S):- & \operatorname{pr}(R 1, R), \operatorname{stage}(S), \operatorname{not} \operatorname{remove}(R 1, S) \\
\operatorname{remove}(R, S 1):- & \operatorname{rule}(R), \text { not } \operatorname{nosource}(R, S), \operatorname{stage}(S), \operatorname{stage}(S 1), \operatorname{lt}(S, S 1) \\
& \operatorname{not} z(R), \operatorname{not} \operatorname{remove}(R, S) . \\
\operatorname{remove}(R, S 1):- & \operatorname{rule}(R), \operatorname{not} \operatorname{nosource}(R, S), \operatorname{stage}(S), \operatorname{stage}(S 1), \operatorname{lt}(S, S 1) \\
& z(R), \operatorname{nbl}(X, R), s(X, S) .
\end{aligned}
$$

According to Step 4, we must add the head $H(r)$ of a generating rule which is to be removed in Step 5, to the set $S$ there. We represent this using a predicate $s(X, S t)$, which informally means that $X$ belongs to set $S$ at stage $S t$, and add the rule:

$$
s(X, S t) \quad:-\operatorname{remove}(R, S t), g(R), \operatorname{head}(X, R) .
$$

Finally, according to Step 2 we have to check whether all rules have been removed in the processing of the graph $G$. This is done by using a predicate removed for the projection of remove to rules and the following rule plus a constraint:

$$
\begin{aligned}
\operatorname{removed}(R) & :-\operatorname{remove}(R, S) . \\
& :-\operatorname{rule}(R), \text { not } \operatorname{removed}(R) .
\end{aligned}
$$


The resulting meta-interpreter program $P_{I_{g}}$ (without $P_{I_{a}}$ ) is shown in Figure 2. Note that $P_{I_{g}}$ is in general also more efficient than $P_{I_{B}}$, since unnecessary totalizations of the partial order can be avoided with $P_{I_{g}}$. By virtue of the results in (Brewka and Eiter 1999) (in particular, Lemma 7.2 there), we can state the following result:

Theorem 3

Let $\mathcal{P}=(P,<)$ be a propositional prioritized program. Then, (i) if $A \in \mathcal{A S}\left(P_{I_{g}} \cup F(\mathcal{P})\right)$ then $\pi(A) \in \mathcal{B P} \mathcal{A S}(\mathcal{P})$, and (ii) for each $A \in \mathcal{B P} \mathcal{A S}(\mathcal{P})$, there exists some $A^{\prime} \in$ $\mathcal{A S}\left(P_{I_{g}} \cup F(\mathcal{P})\right)$ such that $\pi\left(A^{\prime}\right)=A$.

\section{Proof (sketch)}

(i) As in the proof for Theorem 2 we employ the notion of splitting a program (Lifschitz and Turner 1994). Let $Q=P_{I_{g}} \cup F(\mathcal{P})$ and $Q_{a}=P_{I_{a}} \cup F(\mathcal{P})$. Then for each answer set $A$ of $Q$, its restriction $A_{a}$ to the predicates of $Q_{a}$ is an answer set of $Q_{a}$ and $A \in$ $\mathcal{A S}\left(\left(P_{I_{B}} \backslash Q_{a}\right) \cup A_{a}\right)$. By Theorem $1, T=\pi\left(A_{a}\right)=\pi(A)$ is an answer set of $P$.

We can now loosely argue that the deterministic variant of FULL-ORDER with input $T$ creates at most $n$ (where $n=|P|$ ) intermediate values for the set $S$ (not counting the initialization to $\emptyset$ ), referred to as $S_{1}, \ldots, S_{n}$, and implicitly creates (cumulative) sets $R_{1}, \ldots, R_{n}$ of removed rules. It can be seen that there is a one-to-one mapping of rule labels $r_{1}, \ldots, r_{n}$, ordered by the built-in total order $l t$, to $S_{1}, \ldots, S_{n}$ and $R_{1}, \ldots, R_{n}$ via stage. Now, $R_{1}=\left\{r \mid \operatorname{remove}\left(r, r_{1}\right) \in A\right\}$ and $S_{1}=\left\{h \mid s\left(h, r_{1}\right) \in A\right\}$ by the definition of nosource 0 , remove and $s$. We can proceed by induction and assume that $R_{i}=\left\{r \mid \operatorname{remove}\left(r, r_{i}\right) \in A\right\}$ and $S_{i}=\left\{h \mid s\left(h, r_{i}\right) \in A\right\}$ for $1 \leq i<n$. Then, it can be seen that $R_{i+1}=\left\{r \mid \operatorname{remove}\left(r, r_{i+1}\right) \in A\right\}$ and $S_{i+1}=\left\{h \mid s\left(h, r_{i+1}\right) \in A\right\}$ hold by definition of the predicates nosource0, remove and $s$. Observe further that $R_{n}=\{r \mid$ removed $(r) \in A\}$, and that the graph $G$ is empty iff $R_{n}=P$. Since $A$ satisfies the final constraint in $P_{I_{g}}, R_{n}=P$ is guaranteed to hold. Therefore the algorithm outputs "yes" and so $\pi(A) \in \mathcal{B P} \mathcal{A S}(\mathcal{P})$ holds.

(ii) For $A \in \mathcal{B P} \mathcal{A S}(\mathcal{P})$ we can construct an answer set $A^{\prime}$ of $Q$, such that (again by notion of splitting) $A^{\prime \prime}$ is the restriction of $A^{\prime}$ to the predicates defined by $Q_{a}=P_{I_{a}} \cup$ $F(\mathcal{P})$ and $A^{\prime}$ is an answer set of the program $Q_{1}$, which contains $A^{\prime \prime}$ plus all clauses $c$ in Ground $(Q)$ which involve the predicates $g, z$, nosource0, remove, $s$, removed such that $A^{\prime \prime}$ satisfies all literals in $B(c)$ involving predicates defined in $Q_{a} . Q_{1}$ is locally stratified by a stratification defined as follows (where $r_{1}, \ldots, r_{n}$ are the rule identifiers ordered by the built-in total order $l t$ ):

- $\lambda(\operatorname{removed}(r))=2 \times n+1$

- $\lambda\left(\operatorname{nosource}\left(r, r_{i}\right)\right)=2 \times i+1$

- $\lambda\left(\operatorname{remove}\left(r, r_{i}\right)\right)=\lambda\left(s\left(h, r_{i}\right)\right)=2 \times i$

- $\lambda(g(r))=\lambda(z(r))=1$

- $\lambda(a)=0$ for all other atoms $a$ in $Q_{1}$

Since $\pi\left(A^{\prime \prime}\right)=\pi\left(A^{\prime}\right)=A$, it is easy to see that $A^{\prime}$ is an extension to $A^{\prime \prime}$ (which is an answer set of $P_{I_{a}} \cup F(\mathcal{P})$ ) that is fully determined by $\lambda$. $A^{\prime}$ must furthermore satisfy the final constraint in $P_{I_{g}}$. Due to these facts, such an $A^{\prime}$ can be effectively constructed. 


\section{Example 9}

Consider the program in Example 12 and assume priorities $(1)<(3),(2)<(4)$, and $(4)<$ (3). Suppose preferredness of $A_{2}=\{c, \neg d\}$ is checked. Then, the atoms $z(r 1), g(r 2)$, and $g(r 3)$ representing labels are derived, as well as nosource $0(r 4)$ and nosource $0(r 3)$. Both $r 1$ and $r 2$ are sources, but $r 1$ is labeled " $z$ ", so only remove $(r 2, r i)$ and $s(c, r i)$ are derived for $i=1, \ldots, 4$. Thus, nosource $(r i, r 1)$ is derived only for $i=3$. Since $s(c, r 1)$ holds, too, we can derive remove $(r 1, r i)$ and remove $(r 4, r i)$ for $i=2,3,4$. Neither $s(a, r i)$ nor $s(b, r i)$ are derived since $g(r 1)$ and $g(r 4)$ do not hold. Finally, remove $(r 3, r i)$ and $s(\neg d, r i)$ for $i=3,4$ are derived and removed (ri) holds for $i=1, \ldots, 4$, satisfying the final constraint introduced above. Thus, $A_{2}$ is a preferred answer set.

An alternate definition of B-preferred answer sets is provided by (Schaub and Wang 2001); a meta-interpreter program following that definition can be developed using techniques similar to the ones employed in $P_{I_{B}}, P_{I_{g}}$, and the interpreters in the following sections.

\subsection{W-Preferred Answer Sets}

The semantics we have seen in Section 4 is but one way to assign a meaning to prioritized logic programs. In this section we will introduce a related approach due to Wang, Zhou and Lin (2000) following the presentation in (Schaub and Wang 2001).

Definition 7 (W-preferred answer set, $\mathcal{W P A S})$

Let $\mathcal{P}=\left(\left\{r_{1}, \ldots, r_{n}\right\},<\right)$ be a prioritized program. For any set $S \subseteq B_{P}$ of literals, the sequence $S_{i} \subseteq B_{P}(0 \leq i \leq n)$ is defined as follows:

$$
\begin{aligned}
& S_{0}=\emptyset \\
& S_{i}=S_{i-1} \cup\left\{H(r) \mid \begin{array}{ll}
\text { I. } & r \in \mathcal{P} \text { is active wrt. }\left(S_{i-1}, S\right) \text {, and } \\
\text { II. } & \begin{array}{l}
\text { there is no rule } r^{\prime} \in \mathcal{P} \text { with } r^{\prime}<r \text { such that } \\
\text { (a) } r^{\prime} \text { is active wrt. }\left(S, S_{i-1}\right) \text {, and } \\
\text { (b) } H\left(r^{\prime}\right) \notin S_{i-1} .
\end{array}
\end{array}\right\}
\end{aligned}
$$

for all $i=1, \ldots, n$, where a rule $r$ is active wrt. the pair $(X, Y)$ if $B^{+}(r) \subseteq X$ and $B^{-}(r) \cap Y=\emptyset$.

The set $C_{\mathcal{P}}^{W}(S)$ is defined by

$$
C_{\mathcal{P}}^{W}(S)= \begin{cases}S_{n} & \text { if } S_{n} \text { is consistent }, \\ B_{P} & \text { otherwise. }\end{cases}
$$

and $S$ of $\mathcal{P}$ is W-preferred if $C_{\mathcal{P}}^{W}(S)=S$. The set of all W-preferred answer sets of $\mathcal{P}$ is denoted by $\mathcal{W} \mathcal{P} \mathcal{S}(\mathcal{P})$. In this paper we will only consider consistent $\mathrm{W}$-preferred answer sets.

\subsubsection{Adapting the Meta-Interpreter}

In Figure 3 we provide a meta-interpreter for W-preferred answer sets which closely follows Definition 7 and consists of three parts: The first guesses a consistent literal set $A$ (Part 1 below), the second proceeds in stages of rule application according to the definition (Parts 2-8 below), and the final one verifies the "stability" condition $C_{\mathcal{P}}^{W}(A)=A$ (Part 9). 
\% Part 1: Guess a consistent set $\mathrm{S}$.

lit (L) :- head (L,_). lit (L) :- pbl(L,_). lit (L) :- nbl(L,_).

in_S(L) V notin_S(L) :- lit (L).

:- compl $(X, Y)$, in_S $(X)$, in_S $(Y)$.

\% Part 2: Handle preferences.

$\operatorname{pr}(\mathrm{X}, \mathrm{Z}):-\operatorname{pr}(\mathrm{X}, \mathrm{Y}), \operatorname{pr}(\mathrm{Y}, \mathrm{Z})$

$:-\operatorname{pr}(\mathrm{X}, \mathrm{X})$.

\% Part 3: Stage IDs.

stage (S) :- rule (S).

\% Part 4: Evaluate positive bodies.

pos_body_false_S(R) :- rule(R), pbl (X,R), not in_S(X).

pos_body_false_Si(R,Si) :- pbl(L,R), stage(Si), not in_Si(L,Si).

pos_body_false_so(R) :- pbl (L,R).

\% Part 5: Evaluate negative bodies.

neg_body_false_S(R) :- rule $(R), \operatorname{nbl}(X, R)$, in_S (X)

neg_body_false_Si(R,Si) :- nbl(L,R), in_Si(L,Si).

o Part 6: Determine active rules.

active(R,Si) :- rule(R), stage(Si),

not pos_body_false_Si(R,Si), not neg_body_false_S(R).

active_Si(R,Si) :- rule(R), stage(Si), not pos_body_false_S(R), not neg_body_false_Si (R, Si).

active_SO(R) :- rule(R), not pos_body_false_SO(R), not neg_body_false_S(R).

ㅇ Part 7: Check for preferred generating rules.

head_not_in_Si(R,Si) :- stage(Si), head(H,R), not in_Si(H,Si).

preferred_generating_rule_exists(R,Si) :- pr(R1,R), active_Si(R1,Si), head_not_in_Si(R1,Si).

preferred_generating_rule_exists_S0(R) :- pr(R1,R), not pos_body_false_S(R1).

\% Part 8: Compute Si.

in_Si(H,Si) :- head (H,R), $\operatorname{active}(R, S j)$, stage(Sj), stage(Si), lt(Sj,Si), not preferred_generating_rule_exists $(R, S j)$.

in $\mathrm{Si}(\mathrm{H}, \mathrm{Si}):-\operatorname{head}(\mathrm{H}, \mathrm{R})$, active $\mathrm{SO}(\mathrm{R})$, stage $(\mathrm{Si})$

not preferred_generating_rule_exists_so(R).

\% Part 9: Verify "stability".

in_PAS (L) :- in_Si $\left(\mathrm{L}_{-},\right)$.

:- in_PAS $(\mathrm{L})$, not in_S(L)

:- in_S(L), not in_PAS $(L)$.

Fig. 3. Meta-Interpreter $P_{I_{W}}$ for W-Preferred Answer Sets 
Part 1 [Guess a consistent set S] By means of the first three rules we extract all literals occurring in the input program $\mathcal{P}$ into a new predicate lit. Then we guess all possible subsets $S$ of lit by means of the disjunctive rule such that $i n_{-} S(X)$ is true iff $X \in S$. The constraint, finally, ensures that the set $S$ is consistent.

Part 2 [Handle Preferences] To complete the preference relation we transitively close the $p r$ predicate and we also verify that it is irreflexive. The constraint is violated (in that case $P_{I_{W}} \cup F(\mathcal{P})$ admits no answer set) only if a rule is preferred to itself $-\operatorname{pr}(X, X)$.

Part 3 [Stage IDs] Similar to Definition 7, in which we have used the indices $1, \ldots, n$ of the rules $r_{1}, \ldots, r_{n}$, we reuse the IDs of the rules in $\mathcal{P}$ and the arbitrary built-in total order $l t$ over these IDs as IDs of the consecutive stages $S_{1}, \ldots, S_{n}$ of Definition 7 . In this way, the rule IDs and the order $l t$ effectively represent the sequence $S_{1}, \ldots, S_{n}$ without introducing new symbols.

Part 4 [Evaluate positive bodies] According to Definition 7 we need to evaluate the positive and negative bodies of the rules in $\mathcal{P}$ in two ways to verify whether a rule is active wrt. $\left(S_{i}, S\right)$ and $\left(S, S_{i}\right)$, respectively.

The predicates pos_body_false_S $(R)$ and pos_body_false_Si $(R, S i)$ represent the sets of all rules $R$ whose bodies are false according to the set $S$ we have guessed in Part 1 and the set $S_{i}$, respectively, where $S_{i}$ is represented by the predicate $i n_{-} S i$ (with $S_{i}=$ $\left.\left\{L \mid i n_{-} S i(L, S i)\right\}\right)$. pos_body_false $S 0(R)$ covers the base case for $S_{0}=\emptyset$ where the positive body is false w.r.t. $S_{0}$ if some positive body literal exists.

Part 5 [Evaluate negative bodies] Analogously to the case of positives bodies in Part 4, we define predicates which determine the falsity of negative rule bodies w.r.t. $S$ and $S_{i}$. Note that no negative body can be false w.r.t. $S_{0}=\emptyset$, so there is no special predicate for $S_{0}$.

Part 6 [Determine active rules] Now we need to define those rules that are active wrt. $\left(S_{i}, S\right)$ and $(S, S i)$. The former is handled by the first rule, the latter by the second and third rules. Again, the third rule covers the special case for the initial stage $S_{0}=\emptyset$.

Part 7 [Check for preferred generating rules] The rule defining the predicate preferred_generating_rule_exists $(R, S i)$ checks whether a rule, which is preferred to $\mathrm{R}$, exists such that it is active wrt. $\left(S, S_{i}\right)$ and its head does not occur in $S_{i}$. Here we use head_not_in_Si $(R, S i)$ as an auxiliary predicate that checks whether the head of the rule $R$ is in the set $S_{i}$. The third rule once more covers the base case $\left(S, S_{0}\right)=(S, \emptyset)$, where the body of the rule can be simplified.

Part 8 [Compute $S_{i}$ ] To compute $S_{i}$, we have to include the head of all rules which are active wrt. $\left(S_{i-1}, S\right)$ and where no preferred rule exists which is active wrt. $\left(S, S_{i-1}\right)$ and whose head does not already occur in $S_{i-1}$. Also here we need a specialized rule for the base-case where $S_{i-1}=S_{0}$. 
Part 9 [Verify "stability"] Finally, we define a predicate in_PAS as the union of $S_{i}$, for $1 \leq i \leq n$, and check the "stability" condition of Definition 7, i.e., we check whether the relations $i n_{-} S$ and $i n_{-} P A S$ are equal. Any difference between these two will lead to a violation of one of the two constraints and thus a corresponding answer set for $P_{I_{W}} \cup F(\mathcal{P})$ cannot exist.

We provide the following theorem that states the correctness of the meta-interpreter program $P_{I_{W}}$, where $\pi^{\prime}(S)=\{\ell \mid$ in_ $P A S(\ell) \in S\}$.

Theorem 4

Let $\mathcal{P}=(P,<)$ be a propositional prioritized program. Then, (i) if $A \in \mathcal{A S}\left(P_{I_{W}} \cup F(\mathcal{P})\right)$ then $\pi^{\prime}(A) \in \mathcal{W} \mathcal{P} \mathcal{A S}(\mathcal{P})$, and (ii) for each $A \in \mathcal{W} \mathcal{P} \mathcal{A S}(\mathcal{P})$, there exists some $A^{\prime} \in$ $\mathcal{A S}\left(P_{I_{W}} \cup F(\mathcal{P})\right)$ such that $\pi^{\prime}\left(A^{\prime}\right)=A$.

Proof

For the proof of the result, the same techniques as in the proofs of Theorems 2 and 3 can be used. A major difference is that $P_{I_{W}}$ does not start from answer sets generated by $P_{I_{a}}$. Rather, the answer sets of the rules in Part 1 of $P_{I_{W}}$ and $F(\mathcal{P})$, denoted by $P_{1} \cup F(\mathcal{P})$, correspond one to one to the consistent sets $S$ of literals occurring in $P$. Therefore the splitting is done on the literals head, $p b l, n b l, c o m p l, l i t, i n \_S$, notin_S .

As for (i), let $A_{1}^{\mathcal{P}}$ be an answer set of $P_{1} \cup F(\mathcal{P})$ which corresponds to a consistent set of literals $S$ of $P$. We show by induction on $j=1, \ldots, n$ that the sets $S_{j}$ of Definition 7 correspond to the sets $\left\{h \mid i n_{-} S i\left(h, r_{j}\right) \in A_{1^{\prime}}^{\mathcal{P}}\right\}$ where $A_{1^{\prime}}^{\mathcal{P}}$ is the (single) answer set of the rules in Parts 2-8 of $P_{I_{W}}$ and $A_{1}^{\mathcal{P}}$, denoted by $P_{2} \cup A_{1}^{\mathcal{P}}$. Recall that the $r_{i}$ are the rule labels which identify the sets $S_{i}$ : therefore, the $S_{i}$ are totally ordered via the built-in total order $l t$ ("less than") on the rule labels $r_{i}$ by $l t\left(S_{i}, S_{j}\right)$ iff $l t\left(r_{i}, r_{j}\right)$. In what follows, we may assume that $l t\left(r_{i}, r_{j}\right)$ (equivalently, $l t\left(S_{i}, S_{j}\right)$ ) iff $i<j$, i.e., the ordering is given by indices.

To see the correspondence, it can be verified from inspection of the rules in Parts 4 and 5 that active_SO $(r)$ holds, by the third rule of Part 6, exactly for all rules $r$ which are active w.r.t. $(\emptyset, S)=\left(S_{0}, S\right)$ and that preferred_generating_rule_exists_SO $(r)$ holds, by the third rule of Part 7, iff a rule $r^{\prime}$ exists such that $r^{\prime}<r$ and $r^{\prime}$ is active w.r.t. $(S, \emptyset)=\left(S, S_{0}\right)$. Given that $S_{1}$ is the first element in the sequence $S_{1}, \ldots, S_{n}$, the first rule in Part 8 cannot contribute to generate $i n_{-} S i\left(h, r_{1}\right) \in A_{1^{\prime}}^{\mathcal{P}}$. Thus, each such literal must be generated by application of the second rule in Part 8; since $H\left(r^{\prime}\right) \notin \emptyset$ trivially holds for any rule $r^{\prime}$, this means that the conditions I and II in Definition 7 must be satisfied; therefore $h \in S_{1}$ is equivalent to $i n_{-} S i\left(h, r_{1}\right) \in A_{1^{\prime}}^{p}$, which shows the correspondence in the induction base.

Assume now that $S_{j}$ corresponds to $\left\{h \mid i n_{-} S i\left(h, r_{j}\right) \in A_{1^{\prime}}^{p}\right\}$. where $j<n$. Then, we verify that also $S_{j+1}$ corresponds to $\left\{h \mid i n_{-} S i\left(h, r_{j+1}\right) \in A_{1^{\prime}}^{p}\right\}$. First, we observe that for any $i n_{-} S i\left(h, r_{j^{\prime}}\right) \in A_{1^{\prime}}^{p}$, where $j^{\prime} \leq j$, also $i n_{-} S i\left(h, r_{j+1}\right) \in A_{1^{\prime}}^{p}$ holds because of the construction of the rules in Part 8. For literals which do not occur in $S_{j}$ but in $S_{j+1}$, observe that by the induction hypothesis, active_Si $\left(r, s_{j}\right)$ holds, via the first rule in Part 4 and the second rule in Part 5, exactly for rules $r$ which are active w.r.t. $\left(S, S_{j}\right)$, and thus preferred_generating_rule_exists $\left(r, s_{j}\right)$ holds, via the first and second rule of Part 7, iff a rule $r^{\prime}$ exists such that $r^{\prime}<r$ and $r^{\prime}$ is active w.r.t. $\left(S, S_{j}\right)$ and $H\left(r^{\prime}\right) \notin$ $S_{j}$. Furthermore, active $\left(r, s_{j}\right)$ holds, via the second rule in Part 4 and the first rule in 
Part 5, exactly for rules $r$ which are active w.r.t. $\left(S_{j}, S\right)$. Since $i n_{-} S i\left(h, r_{j+1}\right) \in A_{1^{\prime}}^{p}$ such that $i n_{-} S i\left(h, r_{j}\right) \notin A_{1}^{p}$ must be derived by the first rule of Part 8, we thus have that $i n_{-} S i\left(h, r_{j+1}\right) \in A_{1^{\prime}}^{\mathcal{P}}$ and $i n_{-} S i\left(h, r_{j}\right) \notin A_{1^{\prime}}^{\mathcal{P}}$ iff $h \in S_{j+1} \backslash S_{j}$. Therefore, $S_{j+1}$ corresponds to $\left\{h \mid i n_{-} S i\left(h, r_{j+1}\right) \in A_{1^{\prime}}^{p}\right\}$, which completes the inductive argument.

Finally, concerning Part 9, in_PAS encodes $S_{n}$, i.e. $\left\{l \mid\right.$ in_ $\left.P A S(l) \in A_{1^{\prime}}^{\mathcal{P}}\right\}=S_{n}$. The constraints guarantee that in any answer set the criterion $C_{\mathcal{P}}^{W}(S)=S_{n}=S$ is met (recall that inconsistent preferred answer sets are not considered in this paper). In total, we have shown that $A \in \mathcal{A S}\left(P_{I_{W}} \cup F(\mathcal{P})\right)$ implies $\pi^{\prime}(A) \in \mathcal{W} \mathcal{P} \mathcal{A S}(\mathcal{P})$, which proves item (i).

As for (ii), let $Q_{1}$ be the split program obtained from the ground program by dropping rules from Part 1 and $F(\mathcal{P})$ and adding an answer set $A^{\prime \prime}$ of the dropped rules, while keeping only those rules of which the bodies agree with $A^{\prime \prime}$. Then, $Q_{1}$ is (analogous to the program in the proof of Theorem 2) locally stratified. A possible stratification for $Q_{1}$ would be as follows, where $r_{1}, r_{2}, \ldots, r_{n}$ are the rule IDs ordered by $l t$ :

- $\lambda($ in_PAS $(l))=3 \times n+2$

- $\lambda\left(\right.$ preferred_generating_rule_exists $\left.\left(r, r_{i}\right)\right)=3 \times i+2$

- $\lambda\left(\operatorname{active}\left(r, r_{i}\right)\right)=3 \times i+2$

- $\lambda\left(\right.$ head_not_in_Si $\left.\left(r, r_{i}\right)\right)=3 \times i+1$

- $\lambda\left(\right.$ active_Si $\left.\left(r, r_{i}\right)\right)=3 \times i+1$

- $\lambda\left(\right.$ pos_body_false_Si $\left.\left(r, r_{i}\right)\right)=3 \times i+1$

- $\lambda\left(\right.$ neg_body_false_Si $\left.\left(r, r_{i}\right)\right)=3 \times i$

- $\lambda\left(i n \__{-} \operatorname{si}\left(h, r_{i}\right)\right)=3 \times i$

- $\lambda($ preferred_generating_rule_exists_SO $(r))=2$

- $\lambda($ pos_body_false_S $(r))=1$

- $\lambda($ active_SO $(r))=1$

- $\lambda(a)=0$ for all other atoms $a$ in $Q_{1}$

Based on this information, we can readily apply the rules of $Q_{1}$ level by level and effectively construct $A^{\prime} \in \mathcal{A S}\left(P_{I_{W}} \cup F(\mathcal{P})\right)$ such that $\pi^{\prime}\left(A^{\prime}\right)=A$. Specifically, we can verify that $Q_{1}$ has the answer set $A^{\prime}$ such that, for each $j \in\{1, \ldots, n\}$, in_Si $\left(h, S_{j}\right) \in A^{\prime}$ holds iff $h \in S_{j}$ holds and thus $i n_{-} P A S(h) \in A^{\prime}$ iff $h \in A$ holds, so $\pi^{\prime}\left(A^{\prime}\right)=A$.

\subsection{D-Preferred Answer Sets}

Another way to assign a meaning to prioritized logic programs has been introduced by Delgrande, Schaub and Tompits (2000a). For our presentation we again follow (Schaub and Wang 2001).

Definition $8(D$-preferred answer set, $\mathcal{D} \mathcal{P} \mathcal{A S})$

Let $\mathcal{P}=\left(\left\{r_{1}, \ldots, r_{n}\right\},<\right)$ be a prioritized program. For any set $S \subseteq B_{P}$ of literals, the sequence $S_{i} \subseteq B_{P}(0 \leq i \leq n)$ is defined as follows:

$$
\left.\begin{array}{l}
S_{0}=\emptyset \\
S_{i}=S_{i-1} \cup\left\{H(r) \mid \begin{array}{ll}
\text { I. } & r \in \mathcal{P} \text { is active wrt. }\left(S_{i-1}, S\right) \text {, and } \\
\text { II. } & \text { there is no rule } r^{\prime} \in \mathcal{P} \text { with } r^{\prime}<r \text { such that } \\
& \text { (a) } r^{\prime} \text { is active wrt. }\left(S, S_{i-1}\right) \text {, and } \\
\text { (b) } r^{\prime} \notin \operatorname{rule}\left(S_{i-1}\right) .
\end{array}\right.
\end{array}\right\}
$$


for all $i=1, \ldots, n$, where again a rule $r$ is active wrt. the pair $(X, Y)$ if $B^{+}(r) \subseteq X$ and $B^{-}(r) \cap Y=\emptyset$, and rule $(X)$ denotes those rules in $\mathcal{P}$ that have been effectively used to derive literals in $X$.

The set $C_{\mathcal{P}}^{D}(S)$ is defined by

$$
C_{\mathcal{P}}^{D}(S)= \begin{cases}S_{n}, & \text { if } S_{n} \text { is consistent, } \\ B_{P} & \text { otherwise. }\end{cases}
$$

and an answer set $A$ of $\mathcal{P}$ is D-preferred if $C_{\mathcal{P}}^{D}(A)=A$. The set of all D-preferred answer sets of $\mathcal{P}$ is denoted by $\mathcal{D P} \mathcal{P} \mathcal{S}(\mathcal{P})$.

The basic difference between D-preferred and W-preferred answer sets is that the former requires that a higher-ranked rule has been used to actually derive some literal, while for the latter it is sufficient that the literal appears in the head of such a rule.

In fact one can show that the three approaches we have shown get increasingly restrictive in that each approach admits only a subset of the (preferred) answer sets of the previous approach. The following theorem is due to (Schaub and Wang 2001):

Theorem 5 (Schaub \& Wang, 2001)

Let $\mathcal{P}=(P,<)$ be a propositional prioritized program. Then, we have:

$$
\mathcal{D P} \mathcal{A S}(\mathcal{P}) \subseteq \mathcal{W P} \mathcal{A S}(\mathcal{P}) \subseteq \mathcal{B P} \mathcal{A S}(\mathcal{P}) \subseteq \mathcal{A S}(\mathcal{P})
$$

\subsubsection{Adapting the Meta-Interpreter}

The changes from $P_{I_{W}}$ to $P_{I_{D}}$ are relatively small, and we have marked those lines where $P_{I_{D}}$ differs by a vertical bar in Figure 4 .

Instead of tracking literals by means of $i n_{-} S i$ we need to track which rule has been used to derive a particular literal, which is done by means of a predicate in_rule Si $(H, R, S i)$ specifying that in the state denoted by $S i$ the literal $H$ has been derived by means of the rule $R$.

Similarly, we replace head_not_in_Si by a new predicate $r u l e \_n o t \_g e n e r a t i n g \_i n \_S i$ that tells whether a specific rule has been actually applied, not just whether the head of this rule has been derived (possibly from a different rule).

$i n_{-} S i$, finally, is a simple projection of $i n_{-} r u l e_{-} S i$ to obtain the union of all $S_{i}$ s for use in the stability check. We have the following result $\left(\operatorname{recall} \pi^{\prime}(S)=\left\{\ell \mid i n_{-} P A S(\ell) \in S\right\}\right)$.

\section{Theorem 6}

Let $\mathcal{P}=(P,<)$ be a propositional prioritized program. Then, (i) if $A \in \mathcal{A S}\left(P_{I_{D}} \cup F(\mathcal{P})\right)$ then $\pi^{\prime}(A) \in \mathcal{D P} \mathcal{A} \mathcal{S}(\mathcal{P})$, and (ii) for each $A \in \mathcal{D P} \mathcal{A} \mathcal{S}(\mathcal{P})$, there exists some $A^{\prime} \in$ $\mathcal{A S}\left(P_{I_{D}} \cup F(\mathcal{P})\right)$ such that $\pi^{\prime}\left(A^{\prime}\right)=A$.

\section{Proof}

Given that both Definition 8 is very similar to Definition 7 (they differ only in II.b, where $H\left(r^{\prime}\right) \notin S_{i-1}$ is replaced by $r^{\prime} \notin \operatorname{rule}\left(S_{i-1}\right)$, i.e., $r^{\prime}$ has not been used to derive some literal in $S_{i-1}$ ) and that also $P_{I_{W}}$ is very similar to $P_{I_{D}}$, most of the arguments in the proof of Theorem 4 are also valid here. 
Part 1: Guess a consistent set $\mathrm{S}$.

lit (L) :- head (L,_). lit (L) :- pbl(L,_). lit (L) :- nbl(L,_).

in_S(L) V notin_S(L) : - lit (L) .

:- $\operatorname{compl}(\mathrm{X}, \mathrm{Y})$, in_S $(\mathrm{X})$, in_S $(\mathrm{Y})$.

\% Part 2: Handle preferences.

$\operatorname{pr}(\mathrm{X}, \mathrm{Z}):-\operatorname{pr}(\mathrm{X}, \mathrm{Y}), \operatorname{pr}(\mathrm{Y}, \mathrm{Z})$

$:-\operatorname{pr}(\mathrm{X}, \mathrm{X})$.

\% Part 3: Stage IDs.

stage (S) :- rule (S).

\% Part 4: Evaluate positive bodies.

pos_body_false_S(R) :- rule(R), pbl (X,R), not in_S(X).

pos_body_false_Si(R,Si) :- pbl(L,R), stage(Si), not in_Si(L,Si).

pos_body_false_so(R) : - pbl $(\mathrm{L}, \mathrm{R})$.

\% Part 5: Evaluate negative bodies.

neg_body_false_S(R) :- rule $(R), \operatorname{nbl}(X, R)$, in_S(X).

neg_body_false_Si(R,Si) :- nbl(L,R), in_Si(L,Si).

\% Part 6: Determine active rules.

active(R, Si) :- rule(R), stage(Si),

not pos_body_false_Si(R,Si), not neg_body_false_S(R).

active_Si(R,Si) :- rule(R), stage(Si), not pos_body_false_S(R), not neg_body_false_Si(R,Si).

active_SO(R) :- rule(R), not pos_body_false_SO(R), not neg_body_false_S(R).

ㅇ Part 7: Check for preferred generating rules.

rule_not_generating_in_Si(R,Si) :- stage (Si), head (H,R),

| not in_rule_Si(H, R, Si).

| preferred_generating_rule_exists(R,Si) :- pr(R1,R), active_Si(R1,Si),

| $\quad$ rule_not_generating_in_Si(R1,Si).

preferred_generating_rule_exists_S0 (R) :- pr(R1,R), not pos_body_false_S(R1).

- Part 8: Compute Si.

| in_rule_Si(H,R,Si) :- head $(H, R), \operatorname{active}(R, S j), \operatorname{stage}(S j)$, stage $(S i)$,

lt $(S j, S i)$, not preferred_generating_rule_exists $(R, S j)$.

in_rule_Si(H,R,Si) :- head $(\mathrm{H}, \mathrm{R})$, active_SO(R), stage(Si)

not preferred_generating_rule_exists_so(R).

| in_Si(H,Si) :- in_rule_Si $(\mathrm{H}, \ldots, \mathrm{Si})$.

을 9: Verify "stability".

in_PAS (L) :- in_Si $(\mathrm{L},-)$.

:- in PAS $(L)$, not in $S(L)$.

:- in_S(L), not in_PAS $(\mathrm{L})$.

Fig. 4. Meta-Interpreter $P_{I_{D}}$ for D-Preferred Answer Sets 
We just highlight the essential difference: According to the difference between Definition 8 and Definition 7, a predicate $r u l e_{-}$not_generating_in_Si is used in $P_{I_{D}}$ to store the rules which have not been applied to derive some literal in $S_{i}$, rather than head_not_in_Si as in $P_{I_{W}}$, which is defined in the first rule and used in the second rule of Part 7.

For defining rule_not_generating_in_Si, an auxiliary predicate $i n \_r u l e_{-} S i$ is used (defined in Part 8), which is like $i n_{-} S i$ but stores in addition the rule ID $r$ which is used to derive a literal $h$ in a set $S_{i}$. The predicate $i n_{-} S i$ is then defined by a simple projection (third rule of Part 8). Note that the only point where head_not_in_Si is used is in the definition of $r u l e_{-} n o t \_g e n e r a t i n g \_i n \_S i$.

In the inductive step, the argument (in the proof of Theorem 4) that the predicate preferred_generating_rule_exists $\left(r, r_{i}\right)$ holds iff a rule $r^{\prime}$ exists such that $r^{\prime}<r$ and $r^{\prime}$ is active w.r.t. $\left(S, S_{i}\right)$ and $H\left(r^{\prime}\right) \notin S_{i}$, must accordingly be updated such that it reads "preferred_generating_rule_exists $\left(r, r_{i}\right)$ holds iff a rule $r^{\prime}$ exists such that $r^{\prime}<r$ and $r^{\prime}$ is active w.r.t. $\left(S, S_{i}\right)$ and $r^{\prime} \notin \operatorname{rule}\left(S_{i}\right)$."

Apart from these details, the proof remains the same.

\section{Weakly Preferred Answer Sets}

The concept of a weakly preferred answer set relaxes the priority ordering as little as necessary to obtain a preferred answer set, if no answer set is preferred. It can be seen as a conservative approximation of a preferred answer set. So far, this approximation has only been defined for B-preferred answer sets (Brewka and Eiter 1999), though similar extensions to the two other approaches we have seen in Sections 4.3 and 4.4 should be feasible.

\section{Definition 9 (distance)}

Let $<_{1}$ and $<_{2}$ be total orderings of the same finite set $M$. The distance from $<_{1}$ to $<_{2}$, denoted $d\left(<_{1},<_{2}\right)$, is the number of pairs $m, m^{\prime} \in M$ such that $m<_{1} m^{\prime}$ and $m^{\prime}<_{2} m .^{3}$

Clearly, $d\left(<_{2},<_{1}\right)$ defines a metric on the set of all total orderings of $M$. For example, the distance between $a<_{1} b<_{1} c$ and $c<_{2} a<_{2} b$ is $d\left(<_{1},<_{2}\right)=d\left(<_{2},<_{1}\right)=2$. Note that $d\left(<_{1},<_{2}\right)$ amounts to the smallest number of successive switches of neighbored elements which are needed to transform $<_{1}$ into $<_{2}$. This is precisely the number of switches executed by the well-known bubble-sort algorithm.

Definition 10 (preference violation degree, $p v d$ )

Let $\mathcal{P}=(P,<)$ be a prioritized program and let $A \in \mathcal{A S}(\mathcal{P})$. The preference violation degree of $A$ in $\mathcal{P}$, denoted $p v d_{\mathcal{P}}(A)$, is the minimum distance from any full prioritization of $\mathcal{P}$ to any fully prioritized program $\mathcal{P}^{\prime}=\left(P,<^{\prime}\right)$ such that $A$ is a preferred answer set of $\mathcal{P}^{\prime}$, i.e.

$$
\operatorname{pvd}_{\mathcal{P}}(A)=\min \left\{d\left(<_{1},<_{2}\right) \mid\left(P,<_{1}\right) \in \mathcal{F} \mathcal{P}(\mathcal{P}), A \in \mathcal{B P} \mathcal{A S}\left(P,<_{2}\right)\right\} .
$$

The preference violation degree of $\mathcal{P}, p v d(\mathcal{P})$, is defined by $\operatorname{pvd}(\mathcal{P})=\min \left\{p v d_{\mathcal{P}}(A) \mid\right.$ $A \in \mathcal{A S}(P)\}$.

\footnotetext{
3 The definition in (Brewka and Eiter 1999) uses ordinals and deals with possibly infinite $M$. Ours is equivalent on finite $M$.
} 
Now the weakly preferred answer sets are those answer sets which minimize preference violation.

Definition 11 (weakly preferred answer set, $w \mathcal{P A S}$ )

Let $\mathcal{P}=(P,<)$ be a prioritized program. Then, $A \in \mathcal{A S}(P)$ is a weakly preferred answer set of $\mathcal{P}$ iff $p v d_{\mathcal{P}}(A)=p v d(\mathcal{P})$. By $w \mathcal{P} \mathcal{A S}(\mathcal{P})$ we denote the collection of all such weakly preferred answer sets of $\mathcal{P}$.

\section{Example 10}

In the bird \& penguin example, $A_{1}$ is the unique preferred answer set of $\mathcal{P}$. Clearly, every preferred answer set $A$ of any prioritized program $\mathcal{P}$ has $p v d_{\mathcal{P}}(A)=0$, and thus $A$ is a weakly preferred answer set of $\mathcal{P}$. Thus, $A_{1}$ is the single weakly preferred answer set of the program.

\section{Example 11}

Reconsider the program in Example 6. Its answer set $A=\{b\}$ is not preferred. Switching the priorities of the two rules, the resulting prioritized program $\mathcal{P}^{\prime}$ has $C_{A \mathcal{P}^{\prime}}^{B}(A)=\{b\}$, thus $A$ is preferred for $\mathcal{P}^{\prime}$. Hence $\operatorname{pvd}_{\mathcal{P}}(A)=\operatorname{pvd}(\mathcal{P})=1$ and $A$ is a weakly preferred answer set of $\mathcal{P}$.

\section{Example 12}

Consider the following program $\mathcal{P}$ :

$$
\begin{aligned}
& \text { (1) } a \quad:-\operatorname{not} c . \\
& \text { (2) } c:-\operatorname{not} b . \\
& \text { (3) } \neg d:-\operatorname{not} b . \\
& \text { (4) } b:-\operatorname{not} \neg b, a .
\end{aligned}
$$

$\mathcal{P}$ has the answer sets $A_{1}=\{a, b\}$ and $A_{2}=\{c, \neg d\}$. Imposing $(i)<(j)$ iff $i<j$, none is preferred. We have $\operatorname{pvd}_{\mathcal{P}}\left(A_{1}\right)=2$ : (2) and (3) are zombies in the dual reduct which are only defeasible by (4), which must be moved in front of them; this takes two switches. On the other hand, $p v d_{\mathcal{P}}\left(A_{2}\right)=1$ : the single zombie (1) in the dual reduct is defeated if (2) is moved in front of it (here, (4) is a dead rule). Hence, $\operatorname{pvd}(\mathcal{P})=1$, and $A_{2}$ is the single weakly preferred answer set of $\mathcal{P}$.

\subsection{Adapting the Meta-Interpreter for Weakly Preferred Answer Sets}

The transition from an interpreter for preferred answer sets to one for weakly preferred answer sets is simple - just a few clauses have to be added and one has to be slightly altered.

For weakly preferred answer sets, we have to generate a second total ordering (called $p r 1$ ), which does not need to be compatible with the input partial order, and corresponds to $<_{2}$ in Definition 10.

$$
\begin{aligned}
\operatorname{pr} 1(X, Y) \vee \operatorname{pr} 1(Y, X) & :-\operatorname{rule}(X), \operatorname{rule}(Y), X !=Y . \\
\operatorname{pr} 1(X, Z) & :-\operatorname{pr} 1(X, Y), \operatorname{pr} 1(Y, Z) . \\
& :-\operatorname{pr} 1(X, X) .
\end{aligned}
$$




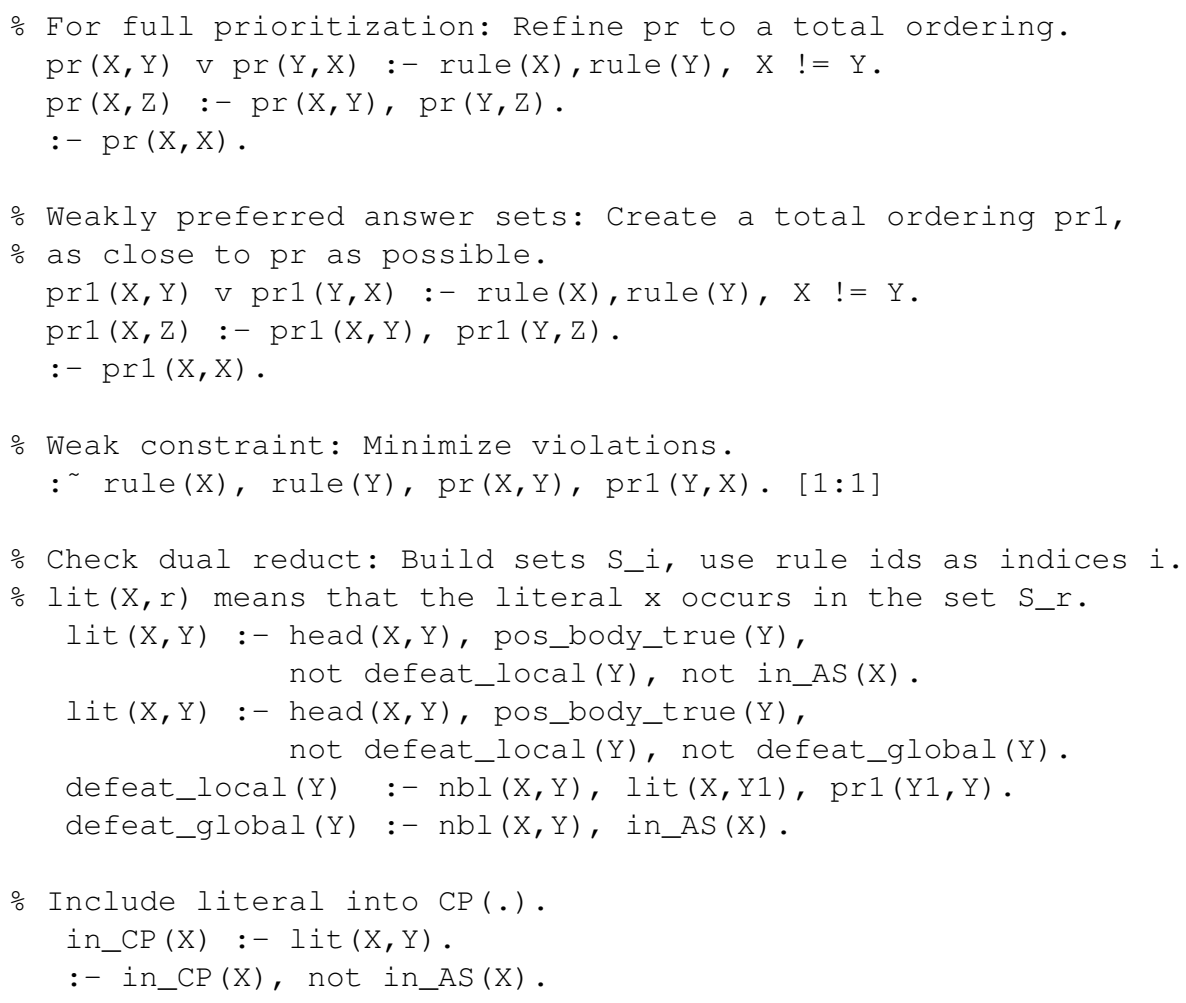

Fig. 5. Meta-Interpreter $P_{I_{\text {weak }}}$ for Weakly Preferred Answer Sets (without $P_{I_{a}}$ )

This ordering should be used to determine the preferred answer sets. Since the given totalization of the input ordering occurs just in one rule of the original program, we just have to update this rule:

$$
\text { defeat_local }(Y) \text { :- } n b l(X, Y), \operatorname{lit}(X, Y 1), \operatorname{pr} 1(Y 1, Y) .
$$

Finally, we want to keep only those orderings which minimize the differences to some totalization of an input ordering. To this end, we state a weak constraint, where each difference in the orderings gets a penalty of one (we don't need the leveling concept here).

$$
: \sim \operatorname{pr}(X, Y), \operatorname{pr} 1(Y, X) .[1: 1]
$$

In this way, each answer set $A$ will be weighted with $p v d_{\mathcal{P}}(A)$, and the optimal answer sets minimize this number, which corresponds exactly to Defs. 9, 10, and 11.

Let us call the resulting interpreter $P_{I_{\text {weak }}}$; a compact listing (without showing $P_{I_{a}}$ ) is given in Figure 5. We have the following result:

\section{Theorem 7}

Let $\mathcal{P}=(P,<)$ be a propositional prioritized program. Then, (i) if $A \in \mathcal{O} \mathcal{A S}\left(P_{I_{\text {weak }}} \cup\right.$ $F(\mathcal{P})$ ), then $\pi(A) \in w \mathcal{P} \mathcal{A} \mathcal{S}(\mathcal{P})$, and (ii) for each $A \in w \mathcal{P} \mathcal{A} \mathcal{S}(\mathcal{P})$, there exists some $A^{\prime} \in \mathcal{O} \mathcal{A S}\left(P_{I_{\text {weak }}} \cup F(\mathcal{P})\right)$ such that $\pi\left(A^{\prime}\right)=A$.

\section{Proof}


Let $Q_{0}$ be the set of all clauses in $P_{I_{\text {weak }}}$ except the two rules defining $p r$, the constraint : $-\operatorname{pr}(X, X)$., and the weak constraint for minimization of violations. After renaming $\operatorname{pr} 1$ to $p r, Q_{0}$ is identical to the meta-interpreter program $P_{I_{B}}$ minus the redundant constraint : $-i n_{-} A S(X)$, not $i n_{-} C P(X)$. Thus, we infer from Theorem 2 that $\mathcal{A S}\left(Q_{0} \cup F((P, \emptyset))\right)$ is in correspondence (i), (ii) as there to $\mathcal{B P} \mathcal{A S}((P, \emptyset))$. Let $Q_{1}$ result from $Q_{0}$ by adding the rules defining $p r$ and the constraint $:-\operatorname{pr}(X, X)$. Then, $\mathcal{A S}\left(Q_{1} \cup F(\mathcal{P})\right)$ is in a similar correspondence to the set of tuples $T=\left\{\left(A,<_{1},<_{2}\right) \mid\left(P,<_{1}\right) \in \mathcal{F} \mathcal{P}(\mathcal{P})\right.$, $\left.A \in \mathcal{B P} \mathcal{A S}\left(P,<_{2}\right),\left(P,<_{2}\right) \in \mathcal{F} \mathcal{P}((P, \emptyset))\right\}$. Adding the weak constraint to $Q_{1}$ (which results in $\left.P_{I_{\text {weak }}}\right)$, we thus have that $\mathcal{O} \mathcal{A S}\left(P_{I_{\text {weak }}} \cup F(\mathcal{P})\right)$ is in similar correspondence to the set $T^{\prime}=\left\{\left(A,<_{1},<_{2}\right) \in T \mid \forall\left(A^{\prime},<_{1}^{\prime},<_{2}^{\prime}\right) \in T: d\left(<_{1}^{\prime},<_{2}^{\prime}\right) \geq d\left(<_{1},<_{2}\right)\right\}$, which in turn naturally corresponds to $w \mathcal{P A S}(\mathcal{P})$. More precisely, we can conclude that for each $A \in \mathcal{O} \mathcal{A S}\left(P_{I_{\text {weak }}} \cup F(\mathcal{P})\right)$, there exists some tuple $\left(\pi(A),<_{1},<_{2}\right)$ in $T^{\prime}$, which corresponds to $\pi(A) \in w \mathcal{P} \mathcal{A S}(\mathcal{P})$; conversely, for each $A \in w \mathcal{P} \mathcal{A S}(\mathcal{P})$, there exists some tuple $\left(A,<_{1},<_{2}\right) \in T^{\prime}$, which corresponds to some $A^{\prime} \in \mathcal{O} \mathcal{A S}\left(P_{I_{\text {weak }}} \cup F(\mathcal{P})\right)$ such that $\pi\left(A^{\prime}\right)=A$. This proves items (i) and (ii) of the theorem.

\section{Example 13}

Reconsider Example 6, which has no preferred answer set. $P_{I_{\text {weak }}} \cup F(\mathcal{P})$ has one optimal answer set (with weight 1 in level 1) containing $i n \_A S(b), \operatorname{pr}(r 1, r 2)$, and $\operatorname{pr} 1(r 2, r 1)$, which is consistent with Example 11.

\section{Example 14}

Reconsider Example 12, which does not have any preferred answer set either. $P_{I_{\text {weak }}} \cup$ $F(\mathcal{P})$ has one optimal answer set (having weight 1 in level 1) containing in_AS(c), in $\_A S(\neg d), \operatorname{pr}(r 1, r 2)$, and $\operatorname{pr} 1(r 2, r 1)$, where the pair $(r 1, r 2)$ is the only difference between $p r$ and $p r 1$, consistent with Example 12.

While $P_{I_{\text {weak }}}$ is a straightforward encoding of the definition of weakly preferred answer set, and gives us an executable specification, it is quite inefficient on larger problem instances because of the large search space generated by the possible total orderings $p r$ and $p r 1$. To improve efficiency, we can use a variant of the graph algorithm FULL-ORDER from Section 4.2.2, based on the following observation. We may arrange the vertices which are removed from $G$, in this order, as a common prefix for orderings $<_{1}$ and $<_{2}$ in the definition of $p v d(A)$. We thus need to guess only optimal completions of $<_{1}$ and $<_{2}$ for those rules that remain in $G$ on termination of FULL-ORDER. In particular, if $G$ is empty, then $<_{1}$ and $<_{2}$ coincide and $A$ is a preferred answer set, hence also weakly preferred. The meta-interpreter programs $P_{I_{g}}$ and $P_{I_{\text {weak }}}$ can be combined to another meta-interpreter program for computing weakly preferred answer sets, which conservatively extends the computation of preferred answer sets in the sense that guessing comes only into play if no preferred answer sets exist. However, we do not further discuss this issue here.

\section{Related Work}

Meta-interpretation of answer sets or answer set-like semantics has been considered by other authors as well, in different contexts. We briefly discuss (Gelfond and Son 1997; Marek and Remmel 2001; Delgrande et al. 2000a; Delgrande et al. 2001) which are related to our work. 
Gelfond and Son. In (Gelfond and Son 1997), the idea of meta-interpretation was used to define the semantics of a language $\mathcal{L}_{0}$ for rules with preferences. $\mathcal{L}_{0}$ is a multi-sorted logical language which has constants for individuals, definite rules, and default rules of the form "If $l_{1}, \ldots, l_{m}$ are true, then normally $l_{0}$ is true," functions and relations for the domain as well as special predicates for defining rules and expressing preference. For example, the formula

$$
\operatorname{default}\left(d, l_{0},\left[l_{1}, \ldots, l_{m}\right]\right)
$$

represents a default rule, where $d$ is its name and $\left[l_{1}, \ldots, l_{m}\right]$ is Prolog-like list notation. Informally, it amounts to the rule $l_{0}:-l_{1}, \ldots, l_{m}$, not $\neg l_{0}$ in extended logic programming. Moreover, the language allows to express conflicts between two default rules; both preferences and conflicts can be declared dynamically by means of rules.

The semantics of $\mathcal{L}_{0}$ is then defined in terms of a transformation of any program $P$ in $\mathcal{L}_{0}$ into an extended logic program $t(P)$ whose answer sets are, roughly speaking, cast into answer sets of the program $P$.

However, there are some salient differences w.r.t. the approach of (Gelfond and Son 1997) and the one presented here.

- First and foremost, the semantics of $\mathcal{L}_{0}$ is defined exclusively by means of a metainterpreter, while our approach implements semantics which have been defined previously without meta-interpretation techniques.

- Secondly, the interpretation program in (Gelfond and Son 1997) uses lists for representing aggregations of literals and conditions on them, in particular "for all" conditions. Such lists cannot be used in datalog programs, as arbitrarily deep function nesting is required for the list concept. We avoid these aggregations by using rule identifiers, a traversal mechanism that exploits an implicit ordering, and default negation.

- Thirdly, in our approach we extend a general answer set meta-interpreter, thus clearly separating the representation of answer sets and prioritization. In the meta-interpreter presented in (Gelfond and Son 1997), this distinction is not obvious.

Marek and Remmel. In a recent paper (Marek and Remmel 2001), Marek and Remmel discussed the issue of a meta-interpreter for propositional normal logic programs in the context of the expressiveness of stable logic programming. They describe a function-free normal logic program Meta1, such that on input of a factual representation $e d b_{Q}$ of a 0-2 normal logic program $Q$ (i.e., each clause in $Q$ has 0 or 2 positive body literals), a projection of the answer sets of Meta $1 \cup e d b_{Q}$ is in one-to-one correspondence to the answer sets of $Q$. The representation $e d b_{Q}$ is similar to our representation $F(Q)$, but explicitly records the position of positive body literals. The meta-interpreter Meta1 is similar to ours, but differs from ours in the following respects:

- Firstly, Meta1 encodes a simple guess and check strategy for the computation of a stable model $S$. It contains a pair of unstratified rules which guess for each atom $a$, whether $a$ is in the stable model $S$ or not. The remaining clauses mimic the computation of the minimal model of $Q^{S}$, using a special predicate computed, and constraints check whether $S$ is reconstructed by it. On the other hand, our metainterpreter $P_{I_{a}}$ has no separate guessing and checking parts. Instead, stability of a 
model is effected by the stable semantics underlying the interpreter. Furthermore, $P_{I_{a}}$ uses negation sensible to the structure of the program $Q$, and in essence preserves stratification (cf. Proposition 1).

- Secondly, because of its naive guess and check strategy, Meta1 is highly inefficient for programs $Q$ which can be evaluated easily. In particular, even for positive programs $Q$, Meta1 explores an exponential search space, and computation of the unique stable model of $Q$ may take considerable time. On the other hand, for stratified $Q$ our meta-interpreter program $P_{I_{a}}$ is, after propagation of the input facts, a locally stratified program and can be evaluated efficiently. Loosely speaking, $P_{I_{a}}$ interprets a significant class of computational "easy" logic programs efficiently.

- Thirdly, Meta1 is only applicable for 0-2 normal logic programs. An extension to arbitrary normal logic programs is possible using similar techniques as in this paper, though.

All these considerations suggest the conclusion that the meta-interpreter Meta1 defined in (Marek and Remmel 2001) is more of theoretical interest, which is fully compliant with the goals of that paper.

Delgrande, Schaub, and Tompits. Based on a seminal approach for adding priorities to default logic (Delgrande and Schaub 1997), Delgrande et al. have developed the PLP framework for expressing priorities on logic programs (Delgrande et al. 2000a; Delgrande et al. 2001). In this framework, extended logic programs with preference information $r \prec r^{\prime}$ between rules attached at the object level, are "compiled" into another extended logic program, such that the answer sets of the latter program amount to the preferred answer sets of the original program. The transformation uses a number of control predicates for the application of rules such that rule preferences are respected as intended in the application of the rules for constructing an answer set of a given program. More specifically, control atoms $a p(r)$ and $b l(r)$ state whether a rule $r$ is applied or blocked, respectively, and atoms $o k(r)$ and $r d y(r)$ control the applicability of rules based on antecedent conditions reflecting the given order information. The framework provides the flexibility to modify the standard transformation, such that transformations for different preference semantics can be designed.

The PLP framework significantly differs from our work in the following respects:

- Firstly, PLP does not use a fixed meta-interpreter for evaluating varying programs, given in a format which can be "processed" by a meta-interpreter. Rather, PLP performs a schematic program construction ad-hoc.

- Secondly, PLP aims at a tool for realizing preferences semantics by providing a suite of special predicates and a particular representation formalism. In contrast, our interest is in casting definitions from first principles to extended logic programs, in a way such that we obtain executable specifications. This way, variations of definitions can be experimented with more flexibly.

- Thirdly, similar as (Gelfond and Son 1997), PLP has no obvious separation of answer sets and prioritization. 


\section{Conclusion}

In this paper, we have considered the issue of building experimental prototypes for semantics of extended logic programs equipped with rule preferences, by using the technique of meta-interpretation. In the course of this, we have presented a suite of meta-interpreters for various such semantics, including a simple meta-interpreter for answer set semantics of plain extended logic programs itself. This meta-interpreter has benign computational properties, and can be used as a building block for meta-interpreters of other semantics.

While the focus of this paper has been on a propositional setting, it is possible to extend the techniques that we have presented for handling non-ground programs as well. However, unless function symbols are allowed at the code level (which is currently not the case in DLV), a technical realization is not completely straightforward. Extending our work to deal with such cases, for which the work reported in (Bonatti 2001b; Bonatti 2001a) might prove useful, and creating a DLV front-end for prioritized program evaluation are issues for further work.

We believe that the work that we have presented in this paper provides supportive evidence to the following items.

- Meta-interpretation can be a useful technique for building experimental prototype implementations of knowledge representation formalisms. In particular, we have shown this for some preferences formalisms extending the seminal answer set semantics.

- By the use of answer set programming, it is possible to cast definitions of the semantics of KR-formalisms quite naturally and appealingly into extended logic programs, which then, by usage of answer set programming engines, provide executable specifications. Note that, in this line, You et al. (1999) have shown how inheritance networks can be compiled to logic programs, and that, on the other hand, semantics of logic programs may be used for refining the semantics of inheritance networks.

- Adding optimization constructs to the basic language of extended logic programming, such as weak constraints in DLV and the constructs provided in Smodels (Niemelä et al. 2000), is valuable for elegantly expressing semantics which are defined in terms of optimal values of cost functions. The semantics of weakly preferred answer sets provides a striking example; other examples can be found e.g. in the domain of diagnostic reasoning. Enhancing ASP engines by further constructs and their efficient realization is thus important for increasing the usability of the ASP compilation and meta-interpreter approach.

Furthermore, the techniques and methods, which we have used in the design of the meta-interpreters, in particular the use of ordering relations, may prove useful for other researchers when designing ASP implementations of applications.

In conclusion, it appears that meta-interpretation, which is well-established in Prologstyle logic programming, is also a topic of interest in ASP, whose exploration also provides useful results for core ASP itself. We are confident that future work will provide further evidence for this view. 


\section{Acknowledgments.}

The authors would like to thank their colleagues, the participants of the AAAI 2001 Spring Symposium on Answer Set Programming, and the anonymous reviewers for their comments and helpful suggestions for improvements. This work was supported by the Austrian Science Fund (FWF) under grants P13871-INF, P14781-INF, and Z29-INF and the European Commission under projects IST-2001-37004 WASP and IST-2002-33570 INFOMIX.

\section{References}

BaAder, F. AND Hollunder, B. 1995. Priorities on Defaults with Prerequisite and their Application in Treating Specificity in Terminological Default Logic. Journal of Automated Reasoning 15, 41-68.

BonatTI, P. A. 2001a. Prototypes for Reasoning with Infinite Stable Models and Function Symbols. In Proc. 6th International Conference on Logic Programming and Nonmonotonic Reasoning (LPNMR-01), T. Eiter, W. Faber, and M. Truszczyński, Eds. Number 2173 in LNCS. Springer, 416-419.

Bonatti, P. A. 2001b. Reasoning with Infinite Stable Models. In Proc. Seventeenth International Joint Conference on Artificial Intelligence (IJCAI) 2001. Morgan Kaufmann Publishers, Seattle, WA, USA, 603-610.

Brewka, G. 1994. Adding Priorities and Specificity to Default Logic. In Proceedings JELIA '94. LNAI 838. Springer, 247-260.

Brewka, G. 1996. Well-Founded Semantics for Extended Logic Programs with Dynamic Preferences. Journal of Artificial Intelligence Research 4, 19-36.

Brewka, G. AND Eiter, T. 1999. Preferred Answer Sets for Extended Logic Programs. Artificial Intelligence 109, 1-2, 297-356.

Brewka, G. And Eiter, T. 2000. Prioritizing Default Logic. In Intellectics and Computational Logic - Papers in Honor of Wolfgang Bibel, S. Hölldobler, Ed. Kluwer, 27-45. ISBN 0-79236261-6.

Buccafurri, F., Leone, N., AND Rullo, P. 1997. Strong and Weak Constraints in Disjunctive Datalog. In Proc. 4th International Conference on Logic Programming and Non-Monotonic Reasoning (LPNMR'97), J. Dix, U. Furbach, and A. Nerode, Eds. Number 1265 in Lecture Notes in AI (LNAI). Springer Verlag, Dagstuhl, Germany, 2-17.

Buccafurri, F., LeOne, N., AND Rullo, P. 1999. Semantics and Expressiveness of Disjunctive Ordered Logic. Annals of Mathematics and Artificial Intelligence 25, 3-4, 311-337. Abstract in Proceedings KR '98.

Delgrande, J. And Schaub, T. 1997. Compiling Reasoning With and About Preferences into Default Logic. In Proceedings IJCAI '97. 168-174.

Delgrande, J. And Schaub, T. 2000. Expressing Preferences in Default Logic. Artificial Intelligence $123,1-2,41-87$.

Delgrande, J., Schaub, T., And Tompits, H. 2000a. Logic Programs with Compiled Preferences. In Proc. 14th European Conference on Artificial Intelligence (ECAI'2000), W. Horn, Ed. IOS Press, 392-398.

Delgrande, J., Schaub, T., And Tompits, H. 2000b. Prioritized Default Logic Revisited: A Compilation of Brewka and Eiter's Approach. In Proceedings European Workshop on Logics in Artificial Intelligence - Journées Européennes sur la Logique en Intelligence Artificielle (JELIA 2000), Malaga, Spain, September 29-October 2, 2000, M. Ojeda-Aciego, I. P. de Guzmán, G. Brewka, and L. Moniz Pereira, Eds. Number 1919 in LNCS. Springer.

Delgrande, J., Schaub, T., AND Tompits, H. 2001. plp: A Generic Compiler for Ordered 
Logic Programs. In Proc. 6th International Conference on Logic Programming and Nonmonotonic Reasoning (LPNMR-01), T. Eiter, W. Faber, and M. Truszczyński, Eds. Number 2173 in LNCS. Springer, 411-415.

Eiter, T., Faber, W., Leone, N., Pfeifer, G., And Polleres, A. 2002. Answer Set Planning under Action Costs. In Proc. 8th European Conference on Artificial Intelligence (JELIA), S. Flesca, S. Greco, G. Ianni, and N. Leone, Eds. Number 2424 in Lecture Notes in Computer Science. Cosenza, Italy, 186-197.

Faber, W., Leone, N., And Pfeifer, G. 1999. Pushing Goal Derivation in DLP Computations. In Proc. 5th International Conference on Logic Programming and Nonmonotonic Reasoning (LPNMR'99), M. Gelfond, N. Leone, and G. Pfeifer, Eds. Number 1730 in Lecture Notes in AI (LNAI). Springer Verlag, El Paso, Texas, USA, 177-191.

FABER, W. AND PFEIFER, G. since 1996. DLV homepage. http: / / www. dlvsystem.com/.

Gelfond, M. AND Lifschitz, V. 1991. Classical Negation in Logic Programs and Disjunctive Databases. New Generation Computing 9, 365-385.

Gelfond, M. And Son, T. C. 1997. Reasoning with Prioritized Defaults. In Proc. Workshop of Logic Programming and Knowledge Representation (LPKR '97). Springer, 164-223.

Lifschitz, V. 1996. Foundations of Logic Programming. In Principles of Knowledge Representation, G. Brewka, Ed. CSLI Publications, Stanford, 69-127.

Lifschitz, V. AND Turner, H. 1994. Splitting a Logic Program. In Proc. 11th International Conference on Logic Programming (ICLP'94), P. Van Hentenryck, Ed. MIT Press, Santa Margherita Ligure, Italy, 23-37.

MareK, V. And Remmel, J. 2001. On the Expressibility of Stable Logic Programming. In Proc. 6th International Conference on Logic Programming and Nonmonotonic Reasoning (LPNMR-01), T. Eiter, W. Faber, and M. Truszczyński, Eds. Number 2173 in LNCS. Springer, 107-120.

MareK, V. AND TRUSZCZyŃSKI, M. 1993. Nonmonotonic Logics - Context-Dependent Reasoning. Springer-Verlag.

Niemelë, I., Simons, P., AND SYRJ ÄNEN, T. 2000. Smodels: A System for Answer Set Programming. In Proc. 8th International Workshop on Non-Monotonic Reasoning (NMR'2000), C. Baral and M. Truszczyński, Eds. Breckenridge, Colorado, USA.

Rintanen, J. 1998. Lexicographic Priorities in Default Logic. Artificial Intelligence 106, 221-265.

Sakama, C. And Inoue, K. 2000. Prioritized Logic Programming and its Application to Commonsense Reasoning. Artificial Intelligence 123, 1-2, 185-222.

Schaub, T. AND WANG, K. 2001. A Comparative Study of Logic Programs with Preference. In Proc. Seventeenth International Joint Conference on Artificial Intelligence (IJCAI) 2001. Morgan Kaufmann Publishers, Seattle, WA, USA, 597-602.

WANG, K., ZhOU, L., AND Lin, F. 2000. Alternating Fixpoint Theory for Logic Programs with Priority. In Computational Logic - CL 2000, First International Conference, Proceedings. Number 1861 in Lecture Notes in AI (LNAI). Springer Verlag, London, UK, 164-178.

You, J.-H., Wang, X., , And Yuan, L. Y. 1999. Compiling Defeasible Inheritance Networks to General Logic Programs. Artificial Intelligence 113, 1-2, 247-268.

Zhang, Y. And Foo, N. Y. 1997. Answer Sets for Prioritized Logic Programs. In Proceedings ILPS 97. 69-83. 